\title{
Research Paper \\ Prevalence of Potentially Inappropriate Medications Among Older People in Qom, Iran Based on STOPP Criteria and Its Association With the Health-Related Quality of Life
}

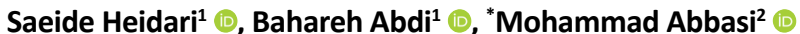

1. Department of Nursing Internal Surgery, School of Nursing and Midwifery, Qom University of Medical Sciences, Qom, Iran. 2. Department of Medical Surgical Nursing, School of Nursing and Midwifery, Iran University of Medical Sciences Tehran Iran.

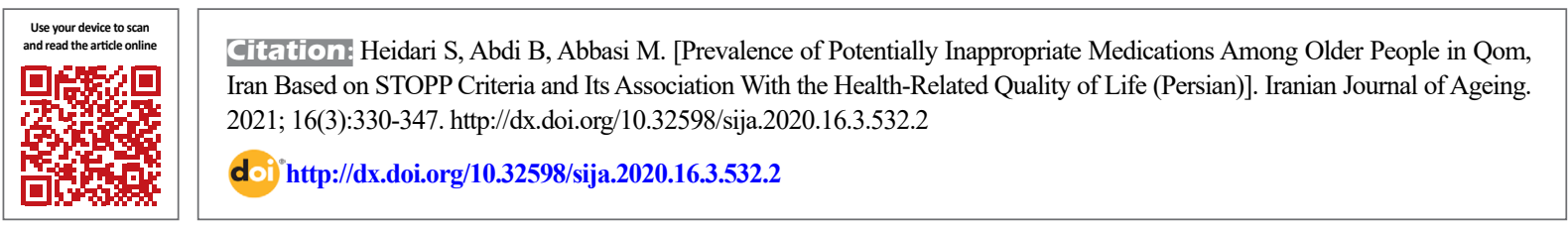

\section{(i) (3)}

Received: 15 Des 2019

Accepted: 17 May 2020 Available Online: 01 Oct 2021

Keywords: Potentially inappropriate medication, elderly, STOPP, Quality of life

\section{A B S TR ACT}

Objectives Aging is associated with an increased prevalence of chronic diseases and multidrug therapy, which increases the risk of drug side effects and changes in quality of life. With the increase of the world's aging proportion, the quality and safety of prescribing drugs has become a global health concern. This study aims to determine the prevalence of Potentially Inappropriate Medications (PIMs) in the elderly in Qom, Iran according to STOPP (Screening Tool of Older Persons' Prescriptions) criteria and its association with their Health-Related Quality of Life (HRQOL).

Methods \& Materials In this cross-sectional and descriptive-analytical study, participants were 783 older patients hospitalized in internal and surgical wards of selected hospitals affiliated to Qom University of Medical Sciences who were selected using a convenience sampling method. The instruments were a demographic/ health information form, Charlson Comorbidity Index (CCI), STOPP version 2, and the 15 D HRQOL questionnaire. The data were analyzed in SPSS v. 20 software using descriptive statistics and logistic regression analysis.

Results The Mean \pm SD number of medications used by the elderly was $7.21 \pm 3.16$, and $79.57 \%$ of patients had multidrug use. The $\mathrm{CCl}$ score of $29.9 \%$ was 4 . The prevalence of PIM was $46.10 \%$ and the most common category of PIM were Drugs that adversely affect those prone to falls (13.79\%), Duplicate Drug Class (12.39\%), Central Nervous System and Psychotropics (5.36\%). The Mean \pm SD score of HRQOL was $0.85 \pm$ 0.12. Logistic regression analysis results showed that $\mathrm{HRQOL}(95 \% \mathrm{Cl}: 0.030-0.307, \mathrm{OR}=0.096)$, multidrug use $(95 \% \mathrm{Cl}$ : 4.780-14.966, $\mathrm{OR}=8.46)$, and $\mathrm{CCl}(95 \% \mathrm{Cl}: 1.36-3.17, \mathrm{OR}=2.079)$ had significant association with PIM use by the elderly $(P<0.001)$.

Conclusion The use of PIM is a common health problem among the elderly and is associated with HRQOL, multidrug use, and CCI. Nurses should evaluate the elderly's medication regimen to identify PIMs.

\section{Extended Abstract}

\section{Introduction}

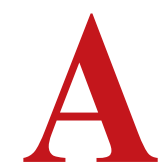

ging is associated with an increased incidence of chronic diseases, multidrug therapy, and thus an increased risk of drug side ef- fects and changes in quality of life. With the increase of the world's aging proportion, the quality and safety of prescribing drugs has become a global health concern. A number of medications or certain doses of some medications can cause side effects in the elderly. Prescribing these medications is

\section{* Corresponding Author:}

\section{Mohammad Abbasi, PhD.}

Address: Department of Medical Surgical Nursing, School of Nursing and Midwifery, Iran University of Medical Sciences Tehran Iran.

E-mail: mohamad_abbasi55@yahoo.com 
potentially inappropriate. One way to identify inappropriate prescriptions and increase the quality of care for the elderly is to recheck the prescribed medications using medication prescription tools by the treatment team, including nurses. One of these criteria is the STOPP (Screening Tool of Older Persons' Prescriptions). Health-Related Quality Of Life (HRQOL) is an important indicator of a person's health status when evaluating health interventions. This study aims to determine the prevalence of Potentially Inappropriate Medication (PIM) in the elderly based on STOPP criteria and assess their subsequent HRQOL.

\section{Methods}

In this descriptive-analytical study, participants were 783 older patients admitted to the internal medicine and surgery wards of three hospitals affiliated to Qom University of Medical Sciences in 2018 who were selected using a convenience sampling method. Inclusion criteria were age $\geq 65$ years, passing of 48-72 hours after hospitalization, daily use of at least one medication, and the ability of to answer questions or having an informed companion. The study obtained an ethical approval from the Ethics Committee of Qom University of Medical Sciences (Code: IR.MUQ. REC.1393.25). Information was collected after obtaining informed consent from the patients and assuring them of the confidentiality of their information through interviews and referring to their medical records. The instruments used included the Charlson Comorbidity Index (CCI), the STOPP Version 2, and the 15D instrument of HRQOL. The CCI predicts 10-year survival in people with several diseases. The STOPP Version 2 contains 80 criteria under 13 categories. The $15 \mathrm{D}$ is self-report tool that assess breathing, mental function, speech, vision, mobility, usual activities, vitality, hearing, eating, elimination, sleeping, distress, discomfort and symptoms, sexual activity, and depression. Its score ranges from 0 to 1 . The content validity of the tools was assessed using the opinions of 10 expert professors and their face validity was assessed using the opinions of 10 older people. Cronbach's alpha value for assessing the scientific reliability of the $15 \mathrm{D}$ was obtained 0.92 ; for the STOPP, it was 0.95. Data were analyzed in SPSS v. 22 software using descriptive statistics, independent t-test and logistic regression analysis to identify the factors affecting the prevalence of PIM (as a dependent variable). The significance level was set at 0.05 .

\section{Results}

The Mean \pm SD age of participates was $72.35 \pm 7.91$ years. Most of them had age 65-75 years $(68.33 \%)$, were women $(52.75 \%)$ and illiterate (81.73\%). Most of them (58.61\%) were hospitalized in the internal wards of the hospital, due to cardiovascular diseases $(25.03 \%)$, respiratory disorders $(16.99 \%)$ and nervous system disorders (16.48\%). The mean number of used medications by the elderly was $7.21 \pm 3.16$ and $79.57 \%$ of them had multidrug use (taking $\geq 5$ medications daily). The CCI score was $29.9 \%$ for four participants and $24.4 \%$ for five participants. The prevalence of PIM based on STOPP criteria was $46.10 \%$. The most common PIMs was benzodiazepines (9.96\%). The most common categories of PIMs were Drugs that adversely affect those prone to falls (13.79\%), Duplicate Drug Class $(12.39 \%)$, Central Nervous System and Psychotropics $(5.36 \%)$ and endocrine system (4.85\%). The Mean \pm SD of total HRQOL score was $0.85 \pm 0.12$; where the lowest score was $0.70 \pm 0.24$ for mobility and $0.79 \pm 0.24$ for breathing, and the highest score was $0.95 \pm 0.11$ for hearing.

The results showed that the total score of HRQOL and its domains (except for hearing, elimination and sexual activity) were significantly different between the two groups of elderly with and without PIM $(\mathrm{P}<0.05)$. Logistic regression analysis results showed that the elderly with no PIM had better overall HRQOL scores than the elderly with PIM. If the HRQOL score increases by 1 unit, the likelihood of prescribing PIM for the elderly decreases by $99.91 \%$ (95\%CI: 0.030-0.307, $\mathrm{OR}=0.096$ ). Those who took $\geq 5$ medications daily were eight times more likely to use PIM than those who took $<5$ medications daily (95\%CI: 4.780-14.966, OR =8.46). Moreover, those with a CCI score of 3-4 were two times more likely to use PIM than those with a CCI score of 1-2 (95\% I : 1.36-3.17, OR =2.079) (Table 1).

\section{Discussion and Conclusion}

PIM use is a common health problem among the elderly in Iran and is associated with HRQOL, multidrug use and CCI. Given the high prevalence of PIM in the elderly and the constant changes in their health status, continuous evaluation of the list of medications used by them for finding PIM should be defined as one of the important nursing measures. One of the reasons for the high prevalence of PIM is the lack of awareness of the treatment team about medication therapy of the elderly, and the tools used to screen PIM prescriptions for the elderly are inappropriate. It is necessary to introduce STOPP criteria in the training programs of the treatment team. One of the limitations of the study was the lack of electronic prescriptions and appropriate software for reviewing prescribed drugs for the elderly and extracting PIM online. It is recommended to design appropriate software for this purpose and to conduct national studies in order to electronize prescriptions for the elderly. 
Table 1. Results of regression analysis for examining the relationship of PIM use with HRQOL, age, CCI, and number of medications

\begin{tabular}{|c|c|c|c|c|c|c|}
\hline \multirow{4}{*}{ Variable } & \multicolumn{4}{|c|}{ Univariate Regression Model } & \multicolumn{2}{|c|}{ Multivariate Regression Model } \\
\hline & \multicolumn{3}{|c|}{ STOPP } & \multirow{3}{*}{$\mathbf{P}$} & \multirow{3}{*}{$\mathbf{P}$} & \multirow{3}{*}{ OR (95\%Cl) } \\
\hline & \multicolumn{3}{|c|}{ Mean \pm SD } & & & \\
\hline & Total & With PIM & Without PIM & & & \\
\hline Mobility & $0.70 \pm 0.24$ & $0.65 \pm 0.23$ & $0.73 \pm 0.24$ & $>0.001$ & $>0.001$ & $0.239(0.437-0.130)$ \\
\hline Vision & $0.85 \pm 0.17$ & $0.83 \pm 0.17$ & $0.86 \pm 0.17$ & 0.037 & 0.038 & $0.402(0.949-0.170)$ \\
\hline Hearing & $0.95 \pm 0.11$ & $0.95 \pm 0.10$ & $0.95 \pm 0.12$ & 0.411 & 0.434 & $0.168(6.11-0.460)$ \\
\hline Breathing & $0.79 \pm 0.24$ & $0.75 \pm 0.23$ & $0.810 \pm 0.24$ & 0.001 & $>0.001$ & $0.324(0.596-0.176)$ \\
\hline Sleeping & $0.82 \pm 0.21$ & $0.76 \pm 0.21$ & $0.86 \pm 0.19$ & 0.001 & $>0.001$ & $0.108(0.222-053.0)$ \\
\hline Eating & $0.83 \pm 0.22$ & $0.79 \pm 0.23$ & $0.85 \pm 0.21$ & 0.001 & $>0.001$ & $0.340(0.647-0.179)$ \\
\hline Speech & $0.91 \pm 0.18$ & $0.89 \pm 0.20$ & $0.92 \pm 0.17$ & 0.048 & 0.042 & $0.448(0.971-0.207)$ \\
\hline Elimination & $0.89 \pm 0.20$ & $0.88 \pm 0.21$ & $0.90 \pm 0.19$ & 0.121 & 0.114 & $0.561(1.148-0.274)$ \\
\hline Usual activities (Self-care) & $0.83 \pm 0.22$ & $0.81 \pm 0.23$ & $0.85 \pm 0.22$ & 0.008 & 0.008 & $0.422(798.0-223.0)$ \\
\hline Mental function & $0.90 \pm 0.18$ & $0.87 \pm 0.19$ & $0.91 \pm 0.17$ & 0.004 & 0.004 & $0.319(0.698-0.146 .0)$ \\
\hline Discomfort and symptoms & $0.93 \pm 0.13$ & $0.91 \pm 0.14$ & $0.94 \pm 0.13$ & 0.025 & 0.019 & $0.278(813.0-095.0)$ \\
\hline Depression & $0.92 \pm 0.14$ & $0.91 \pm 0.16$ & $0.93 \pm 0.13$ & 0.013 & 0.010 & $0.272(0.730-0.102)$ \\
\hline Distress & $0.91 \pm 0.16$ & $0.88 \pm 0.17$ & $0.92 \pm 0.15$ & 0.001 & 0.001 & $0.223(0.54-0.090)$ \\
\hline Vitality & $0.92 \pm 0.15$ & $0.90 \pm 0.16$ & $0.93 \pm 0.14$ & 0.009 & 0.007 & $0.274(0.708-0.106)$ \\
\hline Sexual activity & $0.92 \pm 0.10$ & $0.67 \pm 0.44$ & $0.66 \pm 0.46$ & 0.632 & 0.635 & $1.081(1.493-0.783)$ \\
\hline Total & $0.85 \pm 0.12$ & $0.83 \pm 0.12$ & $0.87 \pm 0.12$ & 0.001 & $>0.001$ & $0.096(0.307-0.030)$ \\
\hline
\end{tabular}

\begin{tabular}{|c|c|c|c|c|c|c|}
\hline & Groups & No. (\%) & No. (\%) & $\mathbf{P}$ & $\mathbf{P}$ & OR(95\%Cl) \\
\hline & $65-75$ & $256(70.91)$ & $279(66.11)$ & & Reference & \\
\hline \multirow[t]{3}{*}{ Age } & $75-85$ & $85(23.55)$ & $103(24.41)$ & 0.175 & 0.598 & $0.912(1.286-0.646)$ \\
\hline & $\geq 85$ & $20(5.54)$ & $40(9.48)$ & & 0.067 & $0.572(1.040-0.314)$ \\
\hline & $1-4$ & $17(4.71)$ & 143(33.88) & & Reference & \\
\hline \multirow[t]{3}{*}{ Number of medications } & & & & 0.001 & & \\
\hline & $\geq 5$ & 344(95.29) & $279(66.12)$ & & 0.001 & $8.46(14.966-4.780)$ \\
\hline & $2-1$ & $45(12.46)$ & $100(0.22)$ & & Reference & \\
\hline \multirow[t]{2}{*}{$\mathrm{CCl}$} & $3-4$ & $217(60.1)$ & $212(51.8)$ & 0.003 & 0.001 & $2.079(3.17-1.36)$ \\
\hline & $\geq 5$ & $99(27.6)$ & $110(26.1)$ & & 0.007 & $1.898(3.034-1.188)$ \\
\hline
\end{tabular}




\section{Ethical Considerations}

\section{Compliance with ethical guidelines}

All ethical principles are considered in this article. The participants were informed about the purpose of the research and its implementation stages. They were also assured about the confidentiality of their information. They were free to leave the study whenever they wished, and if desired, the research results would be available to them.

\section{Funding}

This research did not receive any grant from funding agencies in the public, commercial, or non-profit sectors.

\section{Authors' contributions}

All authors equally contributed to preparing this article.

\section{Conflicts of interest}

The authors declared no conflict of interest. 
This Page Intentionally Left Blank 


\section{مقاله يثوهشى - مقي}

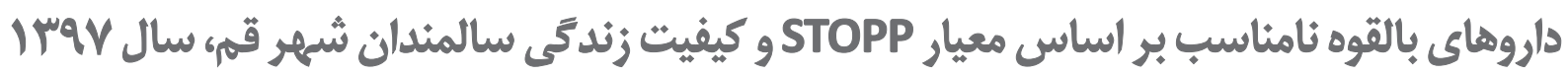

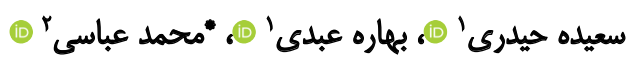

ا. كروه يرستارى داخلى جراحى، دانشكده يرستارى و مامايى، دانشعاه علوم يزٔشكى قهمه قمه، ايران.

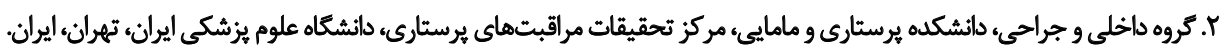

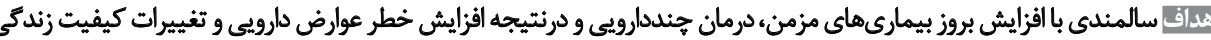

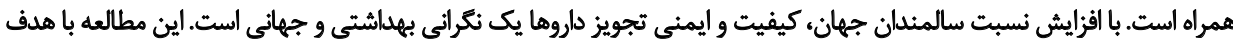

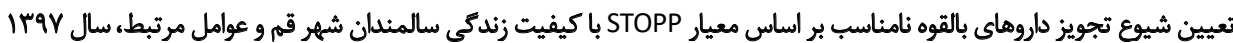

$$
\text { انجام شد. }
$$

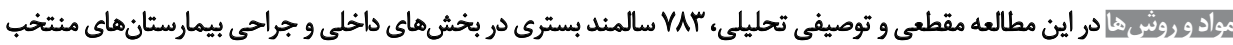

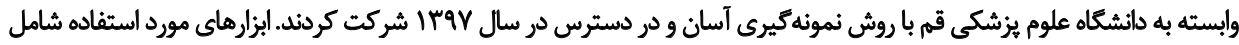

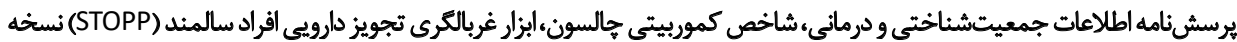

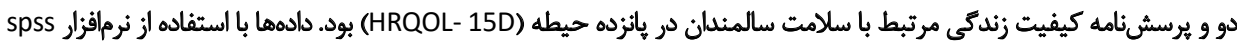

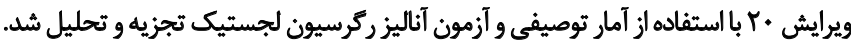

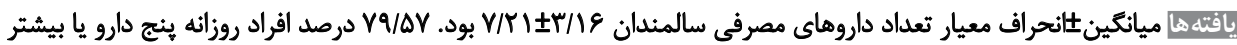

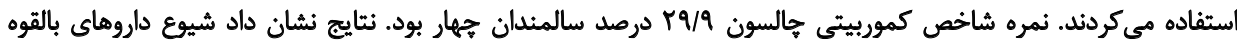

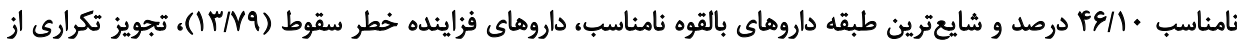

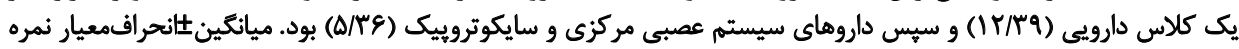

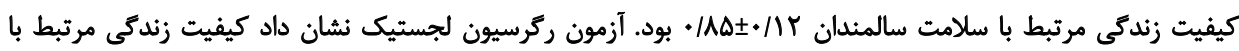

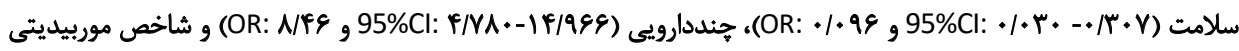

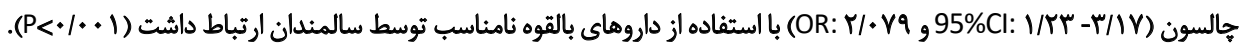

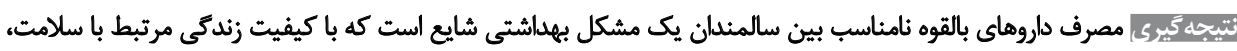

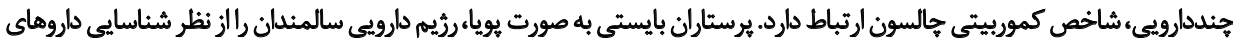

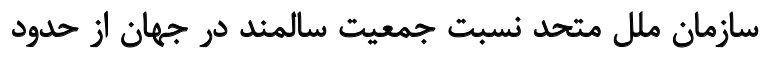

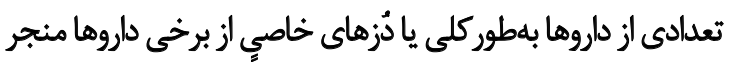

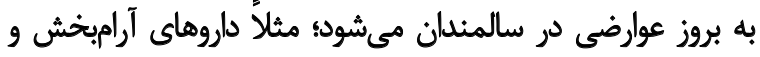

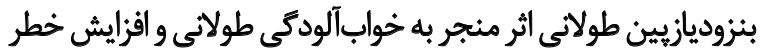

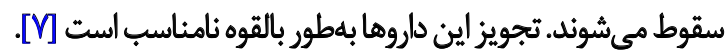

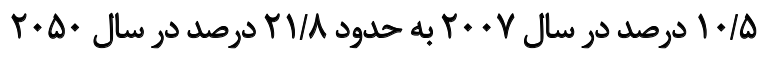

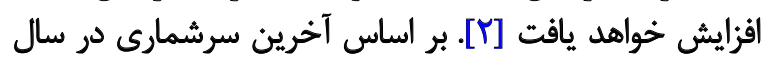

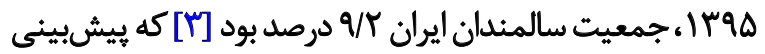

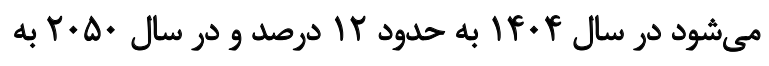

$$
\text { ㄷ... }
$$


بسيارى از مطالعات نشان دادند استفاده از اين معيار ها به شكل

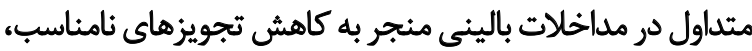

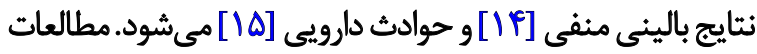

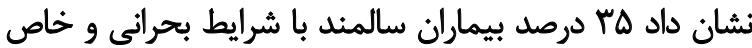

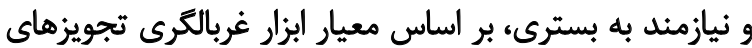

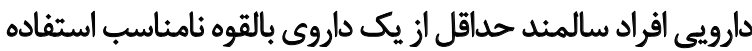

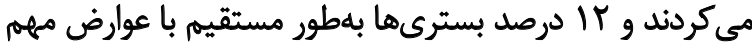

دارويى ارتباط داشت [ه]

مطالعه اوناتاد و همكارانش نشان داد شيوع داروهاى بالقوه

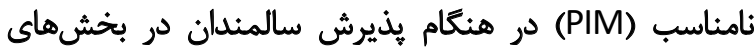

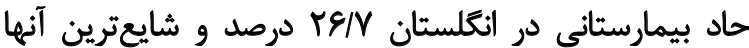

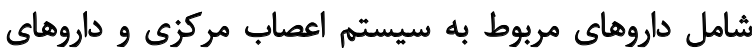

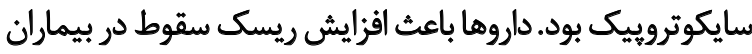
مىشود و بر سيستم ادرارى و تناسلى اثرات منفى داشتند. شيوع داروهاى بالقوه نامناسب هنغام ترخيص از بيمارستان

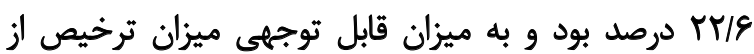

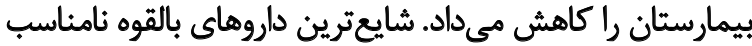

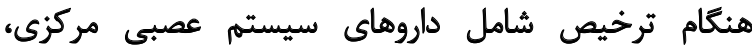

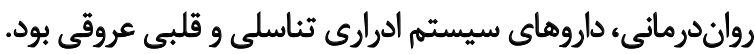

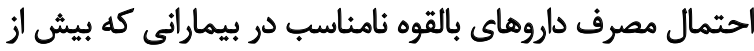

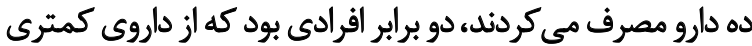

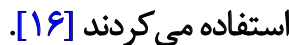

شيوع داروهاى بالقوه نامناسب در مطالعه دالور و همكارانش بردي

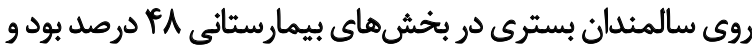

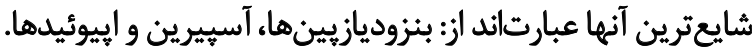

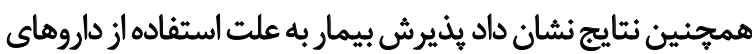

بالقوه نامناسب با سابقه بيمارى قلبى ارتباط داشت [IV [IV].

نتايج مطالعات نشان داد مصرف داروهاى بالقوه نامناسب باعث

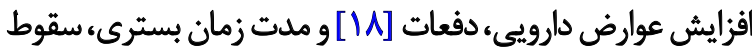

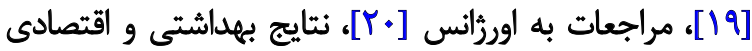

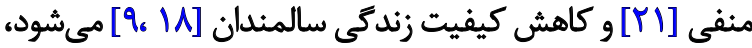

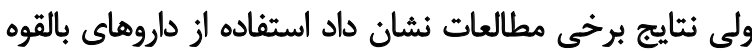

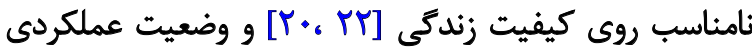

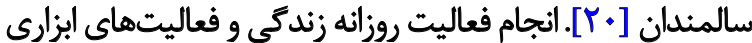

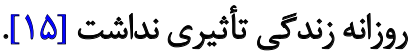

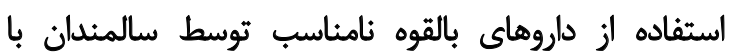

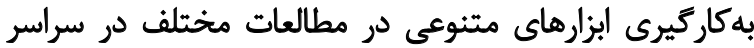

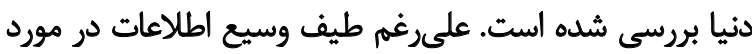

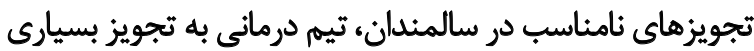

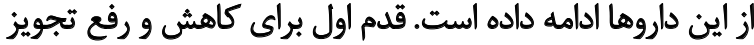

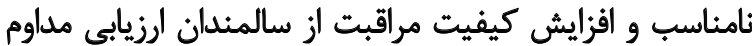

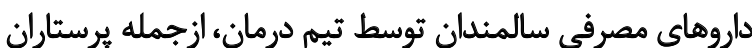

سالمندى با افزايش بروز مشكلات سلامت و بيمارىهاى مزمن

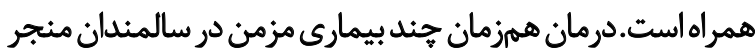

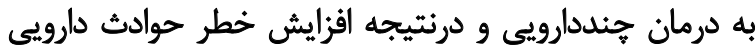

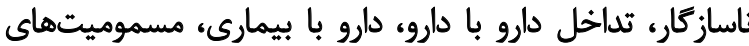

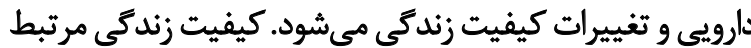

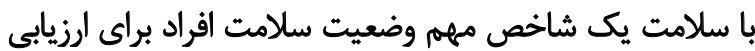

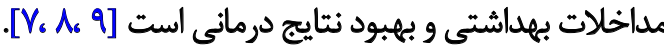
تجويز نامناسب دارويى يك مشكل بهداشتى، جهاتى،

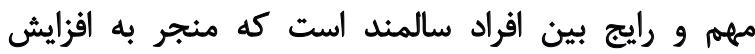

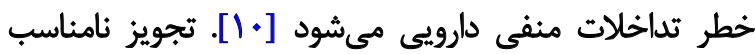

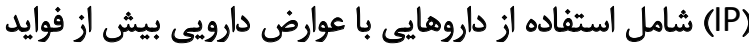

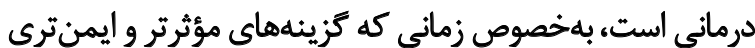

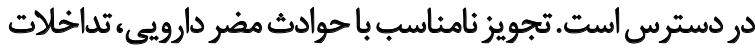
دارويى، بسترى شدنهاي مكرر، بيمارى زايي، مركتومير، اقفزايش

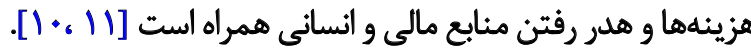

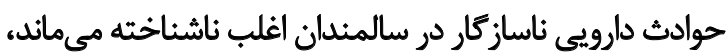

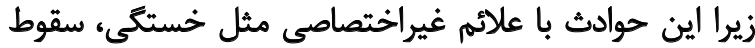

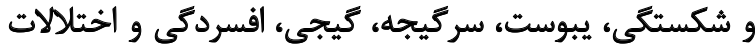

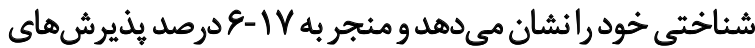

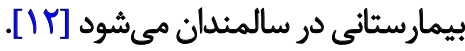
جندارارويى به عنوان مصرف همزمان ينج دارو يا بيشتر به

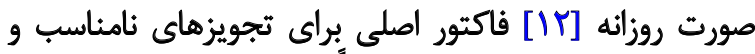

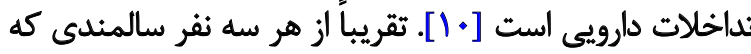

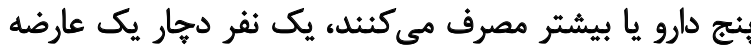

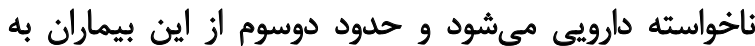

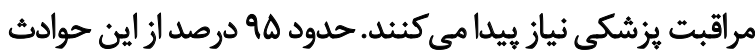

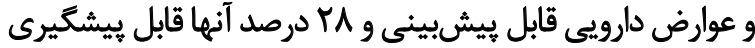

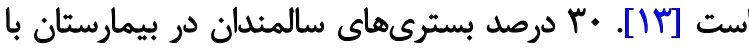
عوارض دارو يا اثرات سمى دارويى ارتباط دارد [V]. به نظر ميرسد، بازنكرى معمول داروهاى بيماران سالمند

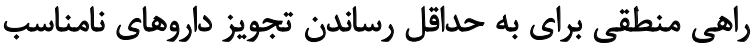

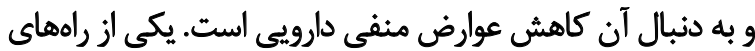

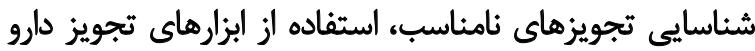

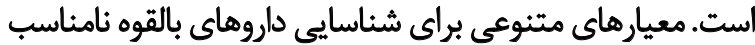

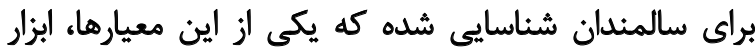

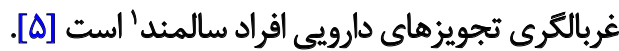
اين شاخص براي بازنكرى فهرست دارويى سالمندان سطح

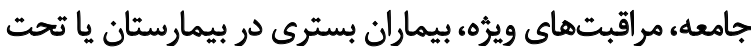

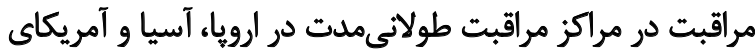

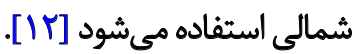

1. Screening Tool of Older Person's Potentially Inappropriate Pre scriptions (STOPP) 
وضعيت تأهل، سطح تحصيلات، بخش بسترى، علت بسترى،

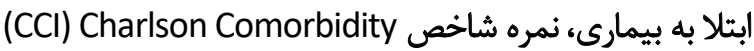

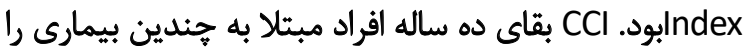

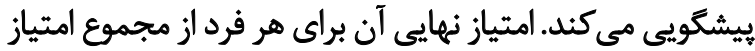

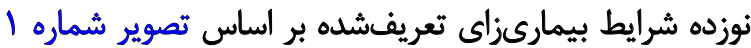

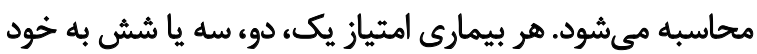

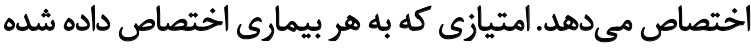
است بر اساس شدت خطرات مرتبط با هر بيمارى است. سه بيمارى ديابت، كبدى و بدخيمىها بر اساس شدت

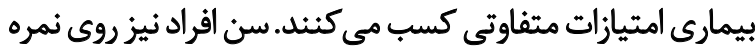

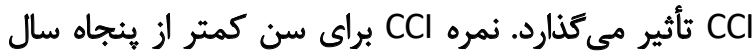

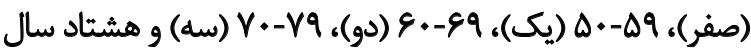

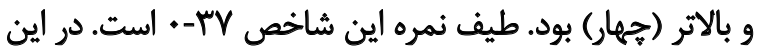

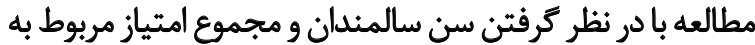

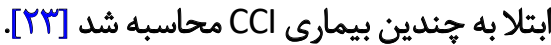
شاخص ابزار غربالكرى تجويزهاى دارويى افراد سالمند بر بران

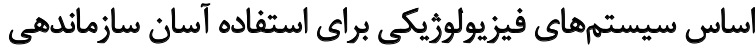

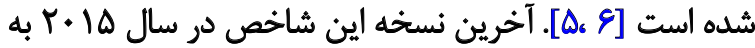

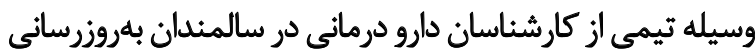

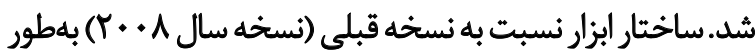

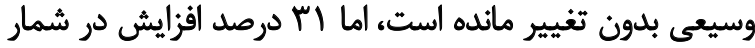

آيتمهاى شاخص وجود داشت [If]

اين شاخص جديد شامل هشتاد آيتم براى تجويز داروها بالقوه

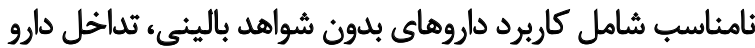

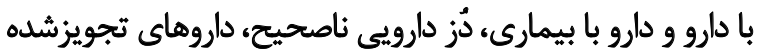

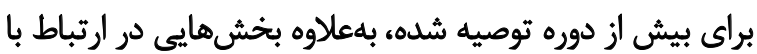

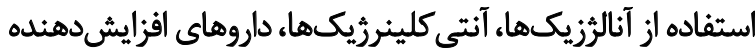

خطر اختلالات شناختى و سقوط است [1T]

شاخص V2 STOPP شامل هشتاد مورد در سيزده بخش

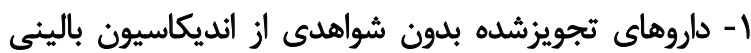

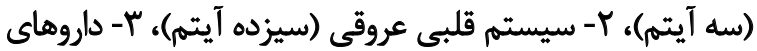

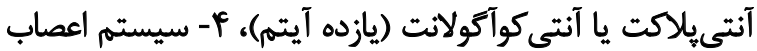

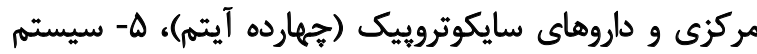

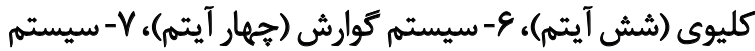

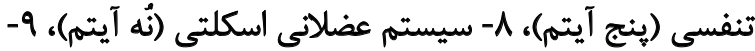

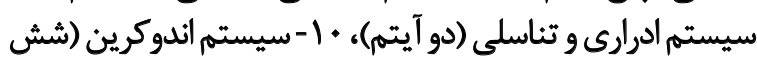

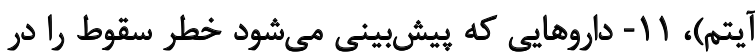

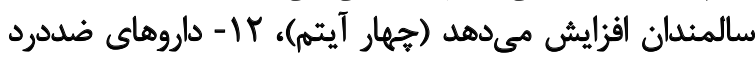

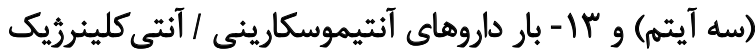

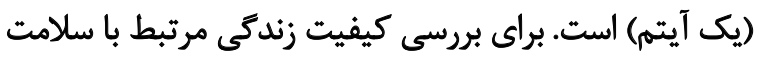

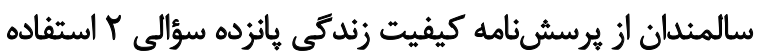

2. Quality of life questionnaire (15D)
برستاران به علت اينكه ارزيابى بيماران را انجام مى دهند، دانش

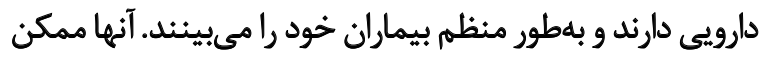

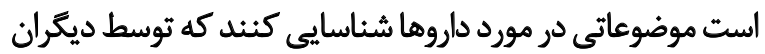

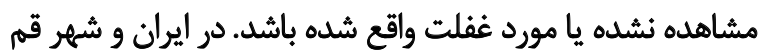

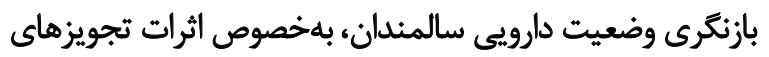

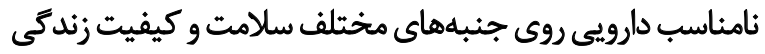

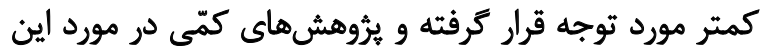

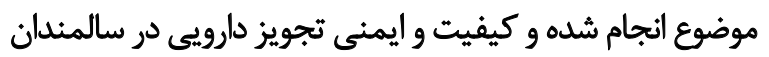
و اثرات تجويز دارويى نامناسب روى سليت سلامت سالمندان همجيجنان يك نكرانى بهداشتى مهرم باقى مانده است.

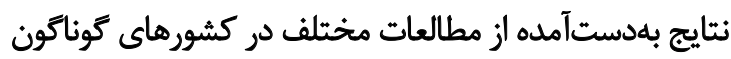

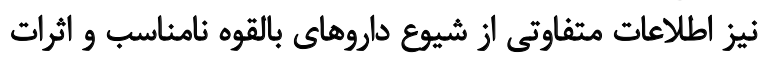

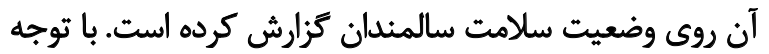
به اينكه ما اطلاعات كمى در مورد شيوع تجويزهائ نامناسب كردي

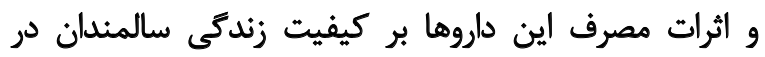

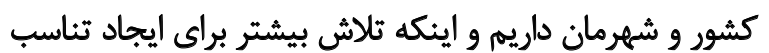

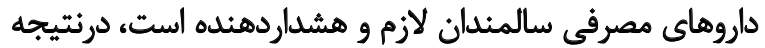

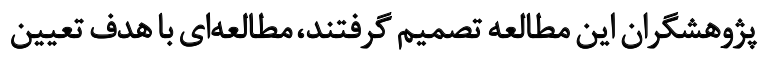

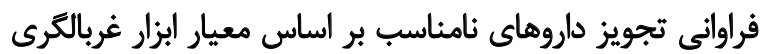

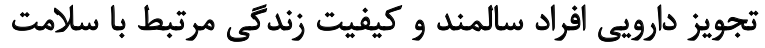

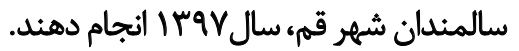

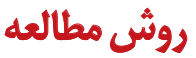

در اين مطالعه توصيفى تحليلى مقطعى VAr سالمند (افراد

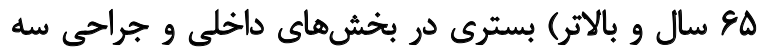

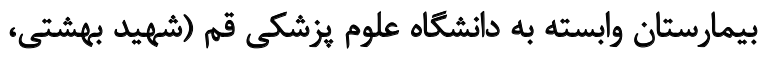

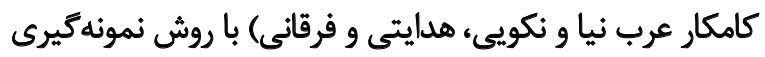

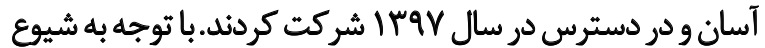

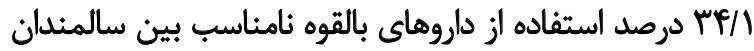

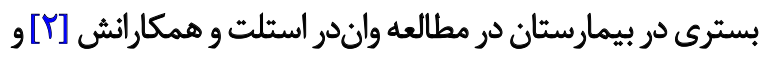

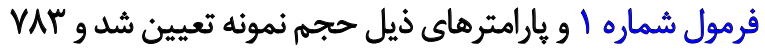
نفر در مطالعه شركت كردند.

$$
\mathrm{n}=\frac{\mathrm{Z}_{1-\mathrm{a} / 2}{ }^{2}(\mathrm{p}(1-\mathrm{p}))}{(\mathrm{d})^{2}}
$$

\section{$a=0 / 05, p=0 / 34, d=0 / 03$}

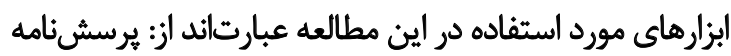

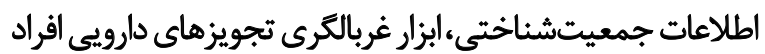

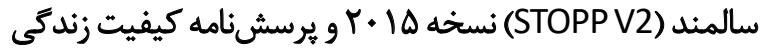
مرتبط با سلامت سالمئدان در هانزده حيطه (HRQOL). يرسشنامه اطلاعات جمعيتشناختى شامل سن، جنس، 
سالمند. معيارهاى خروج نيز شامل عدم تمايل به ادامه مصاحبه

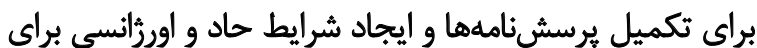

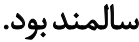

MUQ.) يروهشكر بعد از تصويب طرح، اخذ كد اخلاق

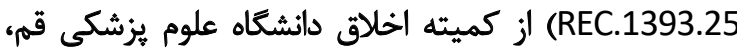

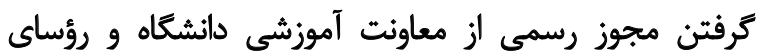

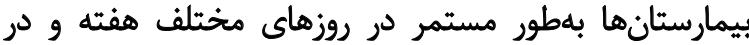

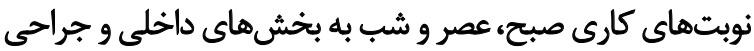

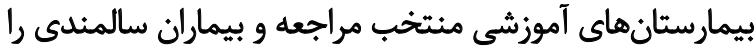

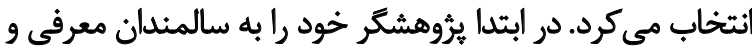

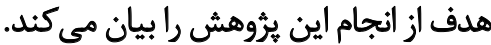

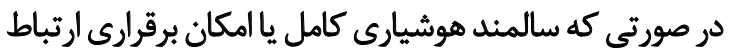

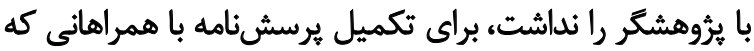

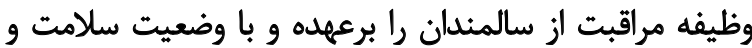

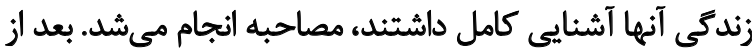

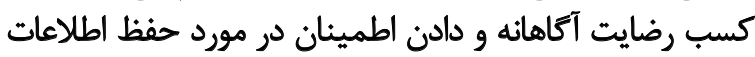

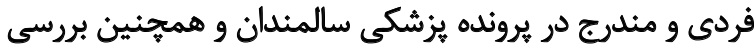

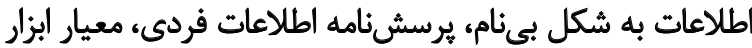

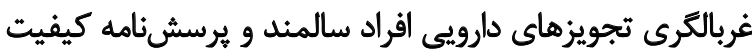

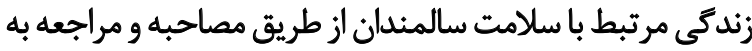

$$
\text { يرونده يزشكى بيماران تكميل شد باند }
$$

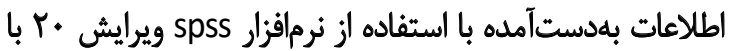

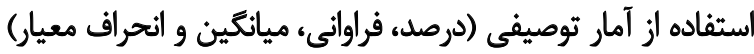
براى توصيف نمونهها و براى شناسايى فاكتورهاى مؤثر بر بر فراواوانى

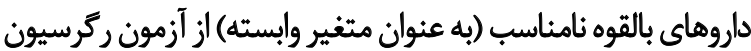

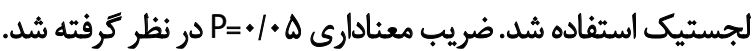

شد. ابزار 15D يك يرسشنامه عمومى، جامع، جندبُعبدى (ياتزده

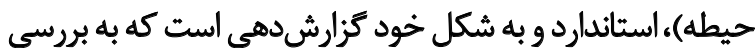

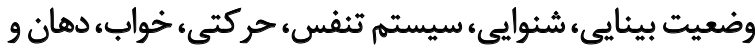

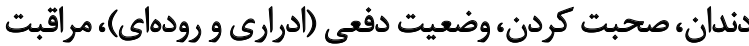

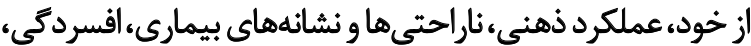
ير يشانى، شور و نشاط و فعاليتهاي جنسيى سالمندان مي ونيردازد. نمره ابزار به دو شكل Single index score نمره شاخص

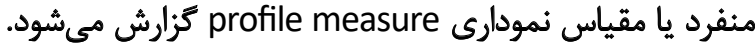

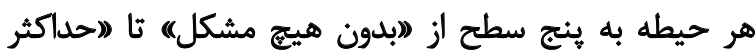

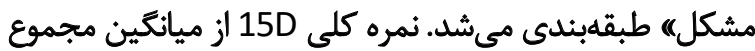

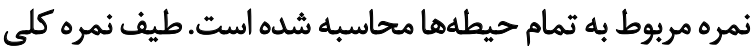

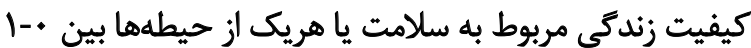

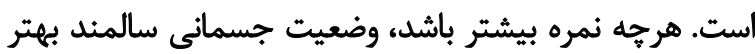

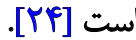

روايي هر دو ابزار، بعد از ترجمه با نظرخواهى از ده نفر از ازيار

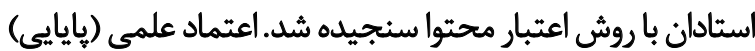

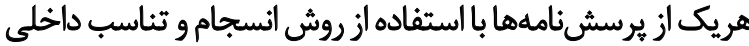

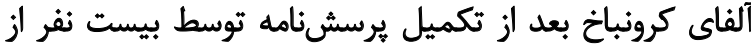

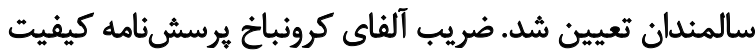

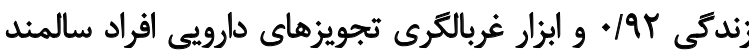

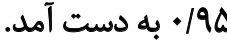
معيارهاى ورود به مطالعه عبارتاند از: سالمندان (افرادى

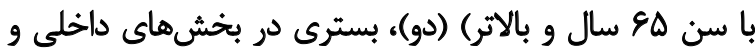

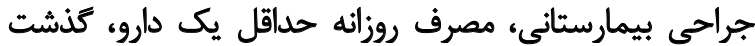

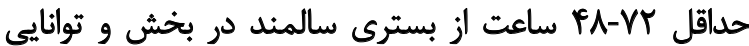

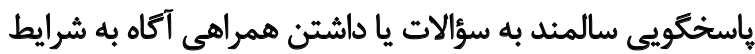

\begin{tabular}{|c|c|}
\hline Comorbidity & Score \\
\hline Myocardial infarction & 1 \\
\hline Congestive heart failure & 1 \\
\hline Peripheral vascular disease & 1 \\
\hline Cerebrovascular disease & 1 \\
\hline Dementia & 1 \\
\hline Chronic pulmonary disease & 1 \\
\hline Rheumatologic disease & 1 \\
\hline Peptic ulcer disease & 1 \\
\hline Mild liver disease & 1 \\
\hline Diabetes without chronic complications & 1 \\
\hline Diabetes with chronic complications & 2 \\
\hline Hemiplegia or paraplegia & 2 \\
\hline Renal disease & 2 \\
\hline Solid tumor & 2 \\
\hline Leukemia & 2 \\
\hline Lymphoma & 2 \\
\hline Moderate or severe liver disease & 3 \\
\hline AIDS/HIV & 6 \\
\hline Metastatic solid tumor & 6 \\
\hline Maximum comorbidity score & 37 \\
\hline
\end{tabular}


جدول ا. توصيف مشخصات جمعيتشناختى و دارويى سالمندان شركت كنثده در مطالعه (n=FAV)

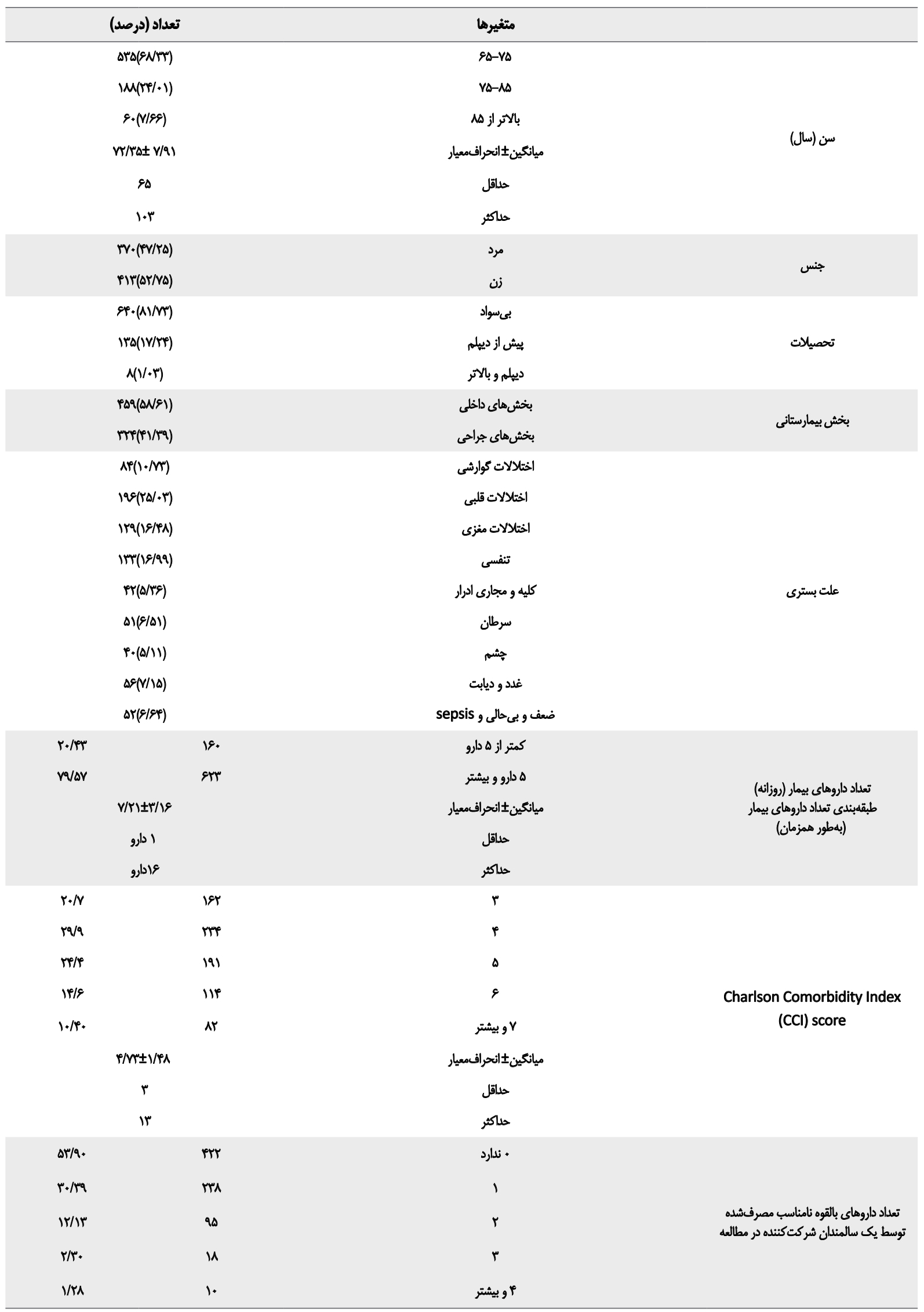


STOPP VY جدول Y. توزيع فراوانى داروهاى بالقوه نامناسب استفادهده توسط سالمندان شركتكنئده در مطالعه بر اساس معيار

\begin{tabular}{|c|c|c|}
\hline تعداد (درصد) & نوع دارو & \\
\hline $9 \vee(1 Y / m q)$ & 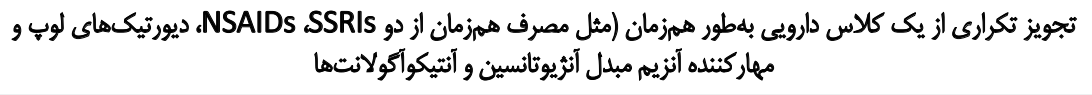 & انديكاسيون دارو \\
\hline$r(r \Delta)$ & بتابلوكرها با براديكاردى (كمتر و مساوى •ه نبض در دقيقه)، بلوك قلبى درجه Y و r & \\
\hline$r(\Delta))$ & ديورتيكهايى لوب به عنوان خط الول درمان در هاييرتنشن & سيستم قلبى عروقى \\
\hline$g(N 9)$ & كل & \\
\hline $1 \cdot(1 / r A)$ & آسيرين با سابقه قبلى زخم ينيتيك بدون مصرف همزمان PPI & \\
\hline$\left.\Delta(8)^{2}\right)$ & NSAIDs با عوامل آنتئيلاكتي بلمطور همزمان بدون PPI بروفيلاكسي & 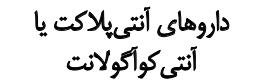 \\
\hline $\mid Q(Y / a T)$ & كل & \\
\hline$g(\mathrm{l})$ & داروهاي ضدافسردكى سه حلقهاي در مبثلايان به دمائس، كلوكوم با زاويه تنكى، آترمالى هلايت قلبي، يروستاثيسم يا سابقه & \\
\hline $11\left(1 / r^{e} \cdot\right)$ & بنزوديازيينها براي بيشتر مساوى حهار هفئه & \\
\hline$\Delta(99)$ & أتنى كلى ترزيكسها و آنتى موسكارينى ها براى درمان عوارض اكستراييراميدال داروهاى نوروليتيك & سيسته عصبي هركزي و \\
\hline$P(\Delta))$ & آتتى كلى نرزئيكها و آنتى موسكارينى ها در بيماران با دليريوم و دمانس & ماروهاى سايكوتروييك \\
\hline $9(1 / / Q)$ & نوروليتيكها به عنوان خواب|آور، مكر اينكه بيمارىهاى خواب به علت سايكوز و دمانس بانشد & \\
\hline$r(\wedge 9)$ & أنتى هيستامينهاي نسل الول & \\
\hline $\operatorname{Pr}\left(\Delta / r^{\varepsilon}\right)$ & كل & \\
\hline$r(Y \Delta)$ & 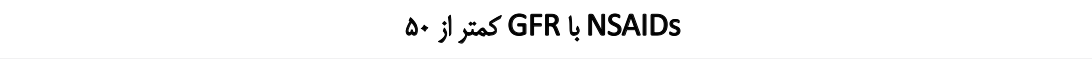 & سيستم كليوي \\
\hline$g(m)$ & براى بيمارى زخم يتيك بلدون عارضه يا ازوفاريت، بيتك زخمى با ذز درمانى كامل براى بيش از هشت هفته & \\
\hline $1 \cdot(1 / r \Lambda)$ & 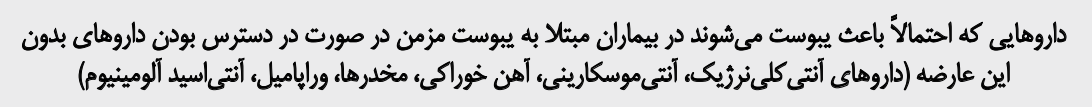 & 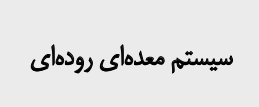 \\
\hline $\mid q(Y / \cdot \Delta)$ & كل & \\
\hline $9(1 / 10)$ & بنزوديازيينها با نارسايى تنفسى مزمن و حاد & 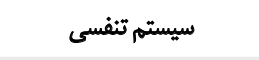 \\
\hline$r(r A)$ & 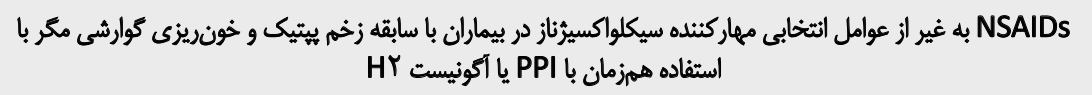 & \\
\hline$r(r \wedge)$ & NSAIDs در بيماران مبتلا به هاييرتنشن شديد يا نارسايى قلبى شديد & سيستم عضلانى - اسكلتى \\
\hline$Y(A 9)$ & NSAIDs انتخابي سيكلواكسيرناز Y همزمان با بيمارى قلبى عروقى & \\
\hline$i r(V / \& \Delta)$ & كل & \\
\hline$r(\Psi A)$ & مصرف داروهاى أتتيموسكارينى در مبتالايان به يروستائيسم مزمن يا دمانس يا نقص شناختى مزمن & 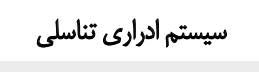 \\
\hline$r q(\varphi / 8 \cdot)$ & سولفين اورةها با عملكرد طولانى (كلى بن كالاميد، كلى مييراميد يا كلرويروياميد) در بيماران مبتلا به ديابت مليتوس نوع r r & \\
\hline$r(r \Delta)$ & تيازوليدنها (روزى كليتازون و ييكليتازون) در بيماران با نارسايى قلبى & 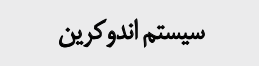 \\
\hline rap(P/AQ) & كل & \\
\hline VA(q/QQ) & بنزوديازيينها & \\
\hline$M(r / s A)$ & داروهاى نوروليتيك & داروهايى كه ييشيينى \\
\hline $9(1 / / Q)$ & داروهاى Z هيينوتيك مثل زولييدم & سالمندان را افزايش مى مدهو نر \\
\hline $1 \cdot A(1 r / M q)$ & كل & \\
\hline$\Delta(94)$ & استفاده منظم از مخلرها بدون استفاده از ملينها & 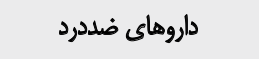 \\
\hline
\end{tabular}




\begin{tabular}{|c|c|c|}
\hline تعداد (درصد) & نوع دارو & \\
\hline$r(19)$ & 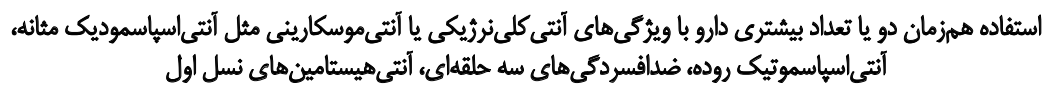 & بار داروهاي آنتىموسكارينى / أنتى كلىريكي \\
\hline rel(F\&/1.) & STOPP VY بر اساس معيار PIM كل & \\
\hline
\end{tabular}

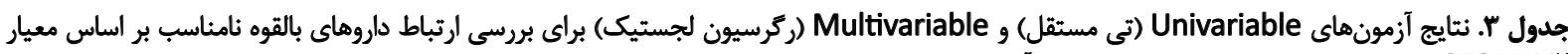

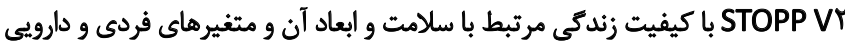

\begin{tabular}{|c|c|c|c|c|c|c|}
\hline \multicolumn{3}{|c|}{ Multivariable Model } & \multicolumn{3}{|c|}{ Univariable Model } & \multirow{4}{*}{ متغيرها } \\
\hline \multirow{3}{*}{ OR (95\%Cl) } & \multirow{3}{*}{$\mathbf{P}$} & \multirow{3}{*}{$\mathbf{P}$} & & STOPP & & \\
\hline & & & \multicolumn{3}{|c|}{ ميانكين \ انحراف معيار } & \\
\hline & & & ندارد PIM & دارد PIM & كل & \\
\hline.$/ \pi r q(\cdot / 1 r+-. / R T V)$ & $>+1 \cdot+1$ & $>+1 .+1$ & $\cdot / r \pm \pm / \pi r$ & $. / 8 \Delta \pm . / \pi$ & $\cdot N \cdot \pm \cdot / T F$ & وضعيت حركتى \\
\hline.$/ r \cdot r(\cdot / / Y \cdot-\cdot / q F q)$ &.$/+\psi \wedge$ &.$/ \cdot \mathrm{rV}$ & $\cdot / N E \pm \cdot / N Y$ & $. / A r \pm . / / V$ & $. / \triangle \Delta \pm . / / V$ & وضعيت بينايى \\
\hline$\cdot / 18 \lambda(\cdot / k 8 \cdot-8 / 11)$ & ./Amp &.$/ 411$ & $. / 9 \Delta \pm+/ 1 r$ & $. / 9 \Delta \pm+/ 1$ & $. / 9 \Delta \pm+/ 11$ & وضعيث شنوايعي \\
\hline.$/ M T P(+/ 1 V E-+/ \Delta Q)$ & $>+1 \cdot+1$ & $.1 . .1$ & $\cdot|\Lambda| \cdot \pm+/ \pi f$ & $\cdot / N \Delta \pm \cdot / \pi$ & $\cdot / M q \pm \cdot / \pi f$ & وضعيت تنفسي \\
\hline$\cdot / \cdot \Lambda\left(\cdot 1 \cdot \Delta T^{H}-/ M T Y\right)$ & $>.1 . .1$ & $.1 . .1$ & $. / 19 \pm+/ 19$ & $\cdot N E \pm+/ M$ & $\cdot|A Y \pm * / Y|$ & وضعيت خواب \\
\hline.$/ M F \cdot(\cdot / 1 V q-\cdot / 9 P V)$ & $>.1 \cdot+1$ & $.1 . .1$ & $\cdot|A \Delta \pm \cdot / T|$ & $. / M \pm \pm \cdot \pi r$ & $. / A r \pm . / M T$ & وضعيت غذا خوردن \\
\hline$+/ 4+\lambda(+/ r+V-+/ 9 V))$ & H H H & $+1 \cdot+\lambda$ & $. / 9 r \pm+/ 18$ & $\cdot / \wedge q \pm+/ Y$ & $\cdot / 9) \pm+/ 11$ & وضعيت صحبت كردن \\
\hline$. / \Delta E \mid\left(\cdot / T V^{2}-1 / \mid P A\right)$ &.$/ 114$ &.$|1 K|$ & $. / 9 \cdot \pm \cdot / 19$ & $\cdot|M \pm \cdot / M|$ & ./А৭ะ•/T. & وضعيت دفعى \\
\hline 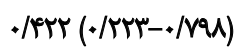 & $.1 \cdot+1$ & $.1 \cdot 1$ & $\cdot / A \Delta \pm \cdot / \pi r$ & $\cdot / \Lambda \mid \pm \cdot / \pi r$ & $\cdot / A r \pm \cdot / M Y$ & فعاليتهاي معمولى (مراقبت از خود) \\
\hline.$/ \% 19(. / 148-. / 894)$ & 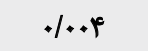 & $.1 .+F$ & $. / 91 \pm \cdot / 17$ & $. / \mathrm{Ar} \pm . / 19$ & $. / 9 . \pm \cdot / 14$ & عملكرد ذهنى \\
\hline.$/ r V \lambda(\cdot / .9 \Delta-. / A 14)$ & $+1+19$ & I.ra & $. / 94 \pm \cdot / 14$ & $. / 91 \pm \cdot / / F$ & $. / q u \pm+/ / H$ & ناراحتى و نشائه بيمارى \\
\hline$\cdot / r r(\cdot / 1 \cdot r-\cdot M r \cdot)$ & .1 .1 & - (1.14 & $. / 94 \pm+/ 1 r$ & $. / 91 \pm+118$ & $. / 97 \pm+/ 17$ & افسرد5ي \\
\hline.$/ M T r(\cdot 1.9 .-. / \Delta f)$ & $.1 .+1$ & $.1 . .1$ & $.19 r \pm \cdot / 10$ & $\cdot / M \pm * / N V$ & $. / 91 \pm * / 18$ & بي بريشائى \\
\hline$\cdot \pi V^{e}(\cdot / 1 \cdot g=\cdot N \cdot 1)$ & $.1 \cdots V$ & .10 .9 & $. / 97 \pm . / 18$ & $. / 9 \cdot \pm \cdot / 18$ & $. / 9 Y \pm . / 10$ & شور و تششاط \\
\hline$V / A)(\cdot / N \wedge r-1 / F q r)$ & . & $.19 \pi \mathrm{TH}$ & $.198 \pm \cdot / 48$ & $. / 8 V \pm . / F F$ & $. / 94 \pm . / 1$ & فعاليث جنسى \\
\hline$\cdot / \cdot q \varepsilon(\cdot 1 \cdot r \cdot-\cdot / r \cdot \eta)$ & $>+1 \cdot .1$ & $.1 \cdot+1$ & $\cdot / A Y \pm=/ I r$ & /Art $\pm+/ M r$ & $. / A \Delta \pm+/ / r$ & نمره كلي 10d \\
\hline \multirow[t]{2}{*}{ OR (Clصده ) } & \multicolumn{2}{|c|}{$\mathbf{P}$} & \multicolumn{2}{|c|}{ فراوانى (درصد) } & طبقات & \\
\hline & reference & & $\operatorname{rrq}(89 / 11)$ & $r \Delta \&(V+191)$ & SQ-VA & \\
\hline.$/ Q 1 T(+/ 949-1 / T N C)$ &.$/ 04$ &.$/ 1 V a$ & $1+r(r f / F I)$ & $\Lambda \Delta(\pi / \Delta \Delta)$ & $A \Delta-V \Delta$ & 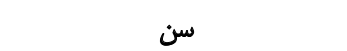 \\
\hline$\cdot / \Delta V T\left(+/ T I F-V / * F_{*}\right)$ & $1.9 V$ & & $f \cdot(q / F \lambda)$ & $r \cdot(\Delta / \Delta F)$ & هـ و بالاتر & \\
\hline \multirow[t]{2}{*}{ NFE (F/VA.-IF/QSE) } & $\begin{array}{c}\text { reference } \\
. / .1\end{array}$ & $.1 . .1$ & $\begin{array}{l}\text { ITT (MT/M) } \\
\text { TV (E\&/IT) }\end{array}$ & $\begin{array}{c}\text { IV }(F / M I) \\
\operatorname{mef}(9 \Delta / T q)\end{array}$ & ه دارو و وايشتر & تعداد داروهاي دريافتى سالمند \\
\hline & reference & & $1 . \cdot(\cdot \pi T)$ & Pa $(\mathrm{IT/Re})$ & $1-r$ & \\
\hline$r / . V A(V / r q-r / M V)$ & $.1 . .1$ & • & $\operatorname{MIr}(\Delta) / A)$ & $\operatorname{riv}(8 . / 1)$ & $r-r$ & $\mathrm{CCl}$ \\
\hline IAQA (M/MA-r/.MT) & $.1 \cdot \cdot v$ & & $11 \cdot(r q /)$ & 99 (TV/P) & هو لهيشتر & \\
\hline
\end{tabular}




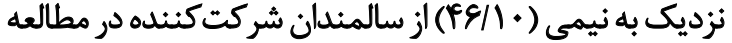
حاضر حداقل يك داروى بالقوه نامناسب (PIM) بر اساس معيار

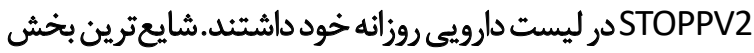

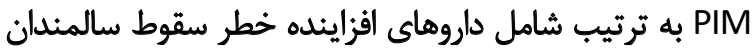

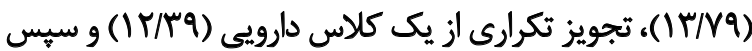

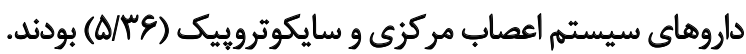

در مطالعه بو و همكارانش با استفاده از ابزار STOPPV2 روي

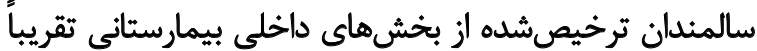

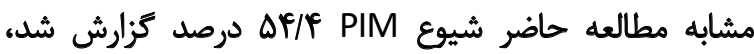

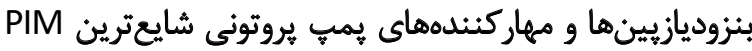

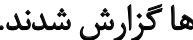

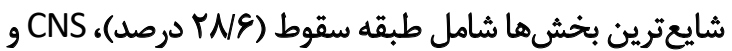

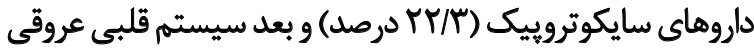

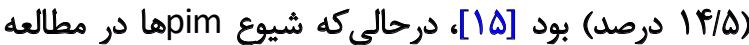

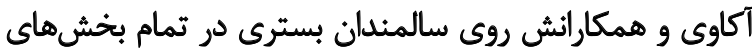

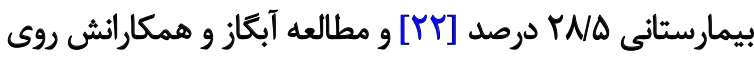

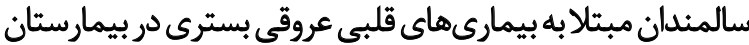

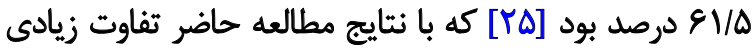

داشت.

در مطالعه آبكاز و همكارانش، شايعترين ACIM دامل

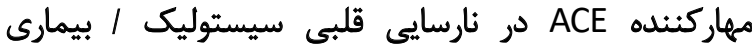

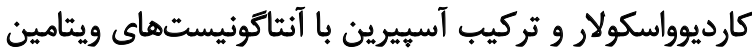

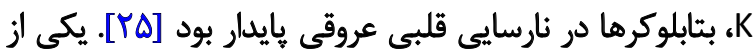

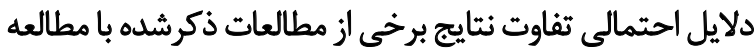

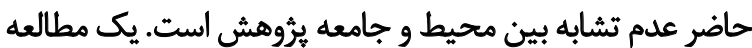

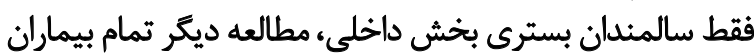

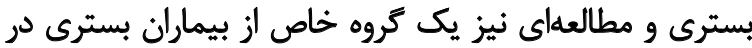
بخش داخلى (بيماران قلبى) را بررسى كرده است.

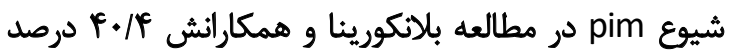

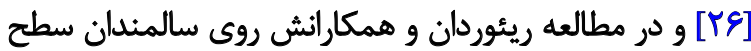

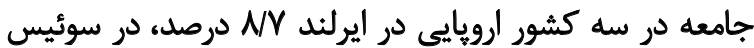

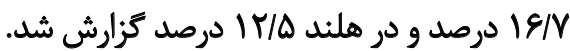

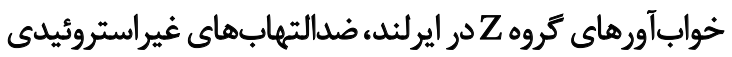

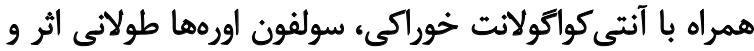

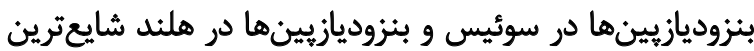

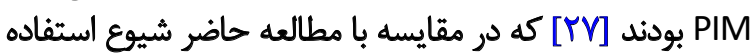

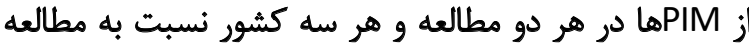

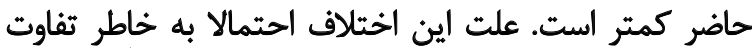

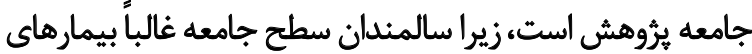

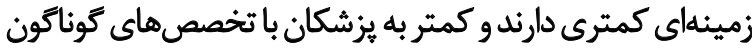

نتايج نشان داد ميانكين سنى سالمندان شركت كنينده در مطالعه

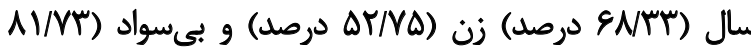

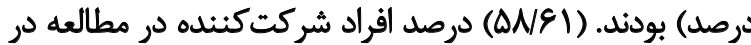

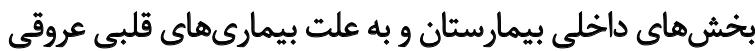

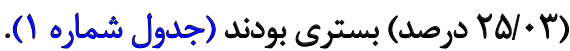

ميانگين و انحراف معيار تعداد داروهاى مصرفى افراد شركتكننده در مطالعه

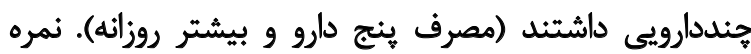
Charlson Comorbidity Index

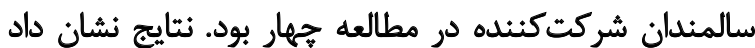

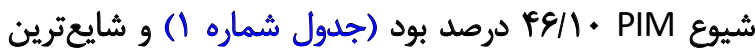

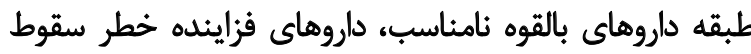

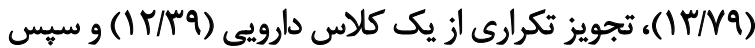

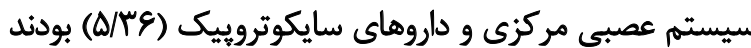

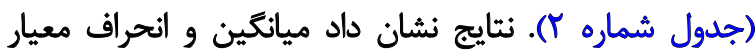

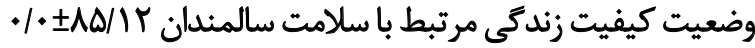

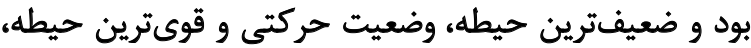
وضعيث شنوايى سالمندان بود (جدول شماره بات). نتايج نشان داد كه درصد و فراوانى استفاده از داروهاي بالقوه

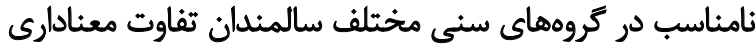
نداشت (P= (P) (جدول شماره مَ).

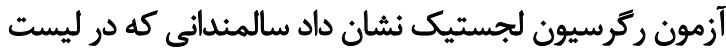

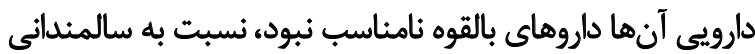

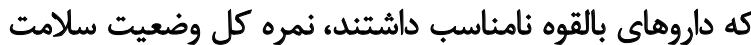

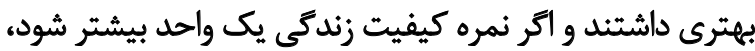
س9/9

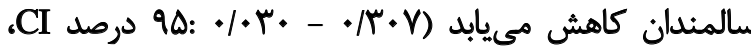

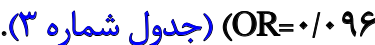
نتايج آزمون ركرسيون لجستيك نشان داد سالمندانى كه

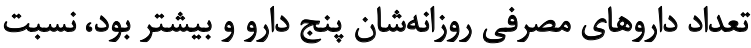

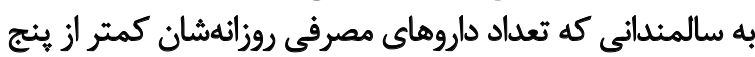

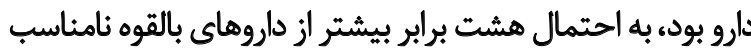

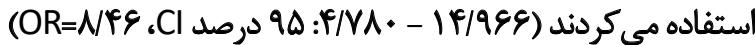

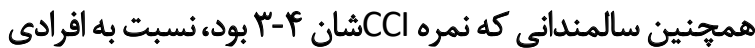

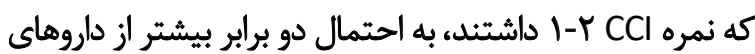

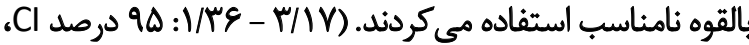

(OR=r/・V9 


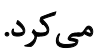

نتايج مطالعه كوهورت آيندهنكر كاهير و همكارانش در مورد

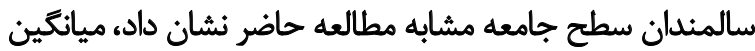
نمره كيفيت زندكى سالمندان بر اساس ابزار

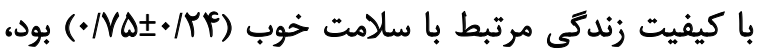

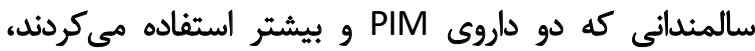

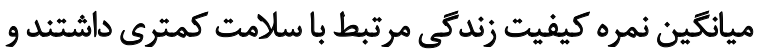

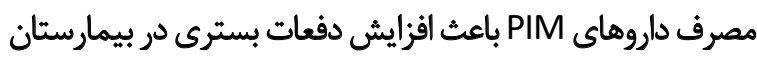

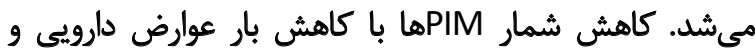

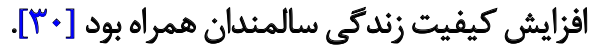

نتايج مطالعه زانك و همكارانش نيز نشان داد، ميانكين

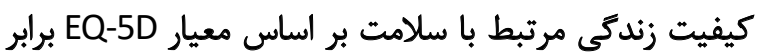

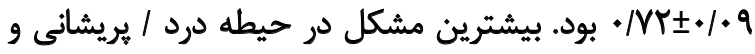

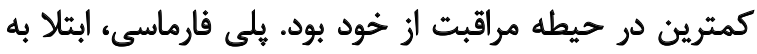

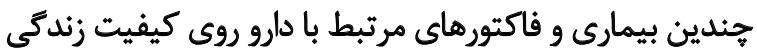
سالمندان مؤثر بود [9]

مطالعه كوهورت كذشتهنكر برون و همكارانش در آمريكا

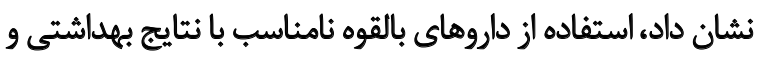

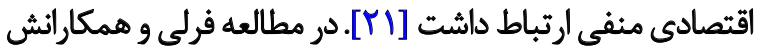

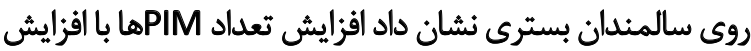

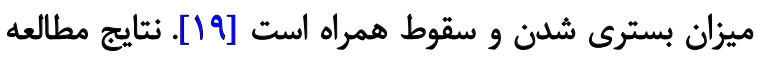

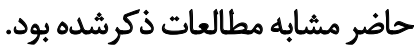

ولى نتايج مطالعه موريارتى و همكارانش در يك مطالعه

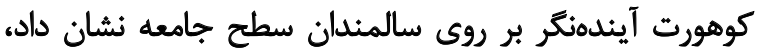

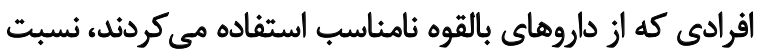

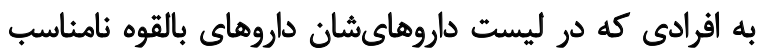

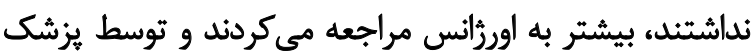

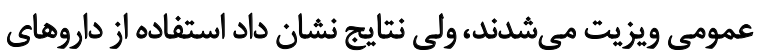

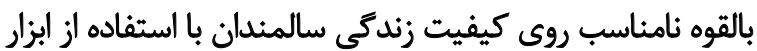

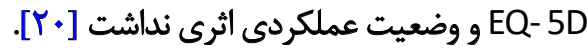

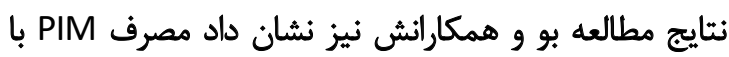

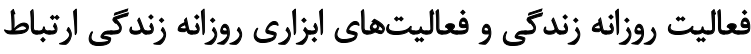

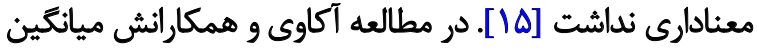

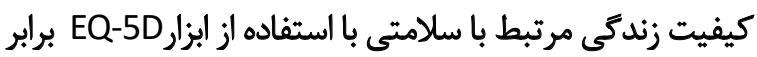

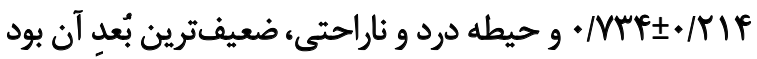

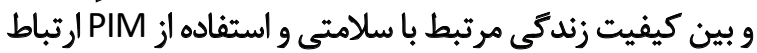

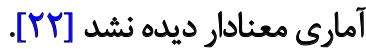

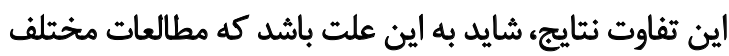

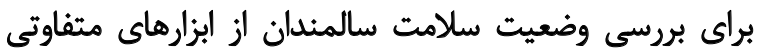

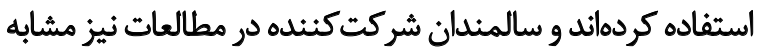

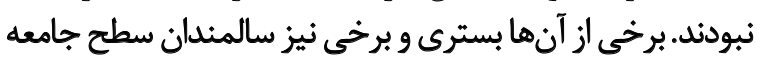

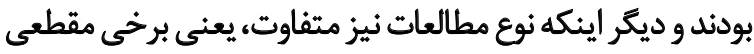

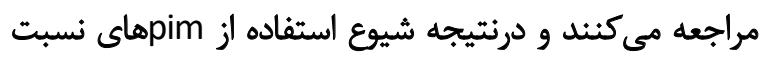

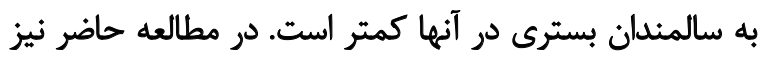

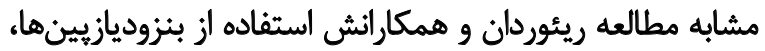

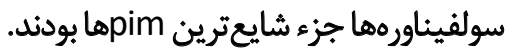

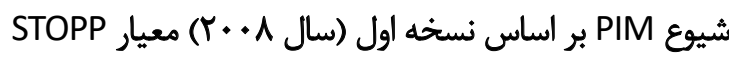

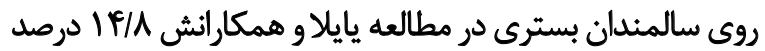

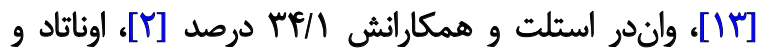

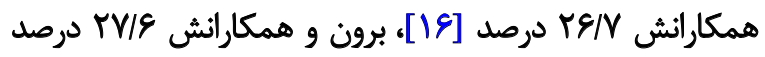

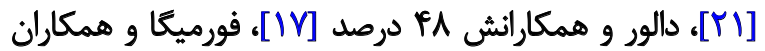

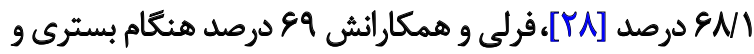

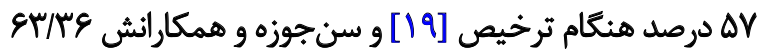

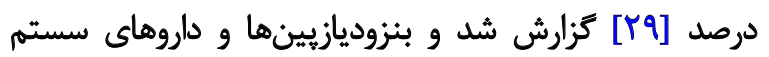

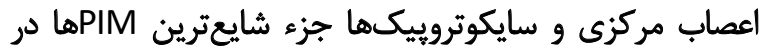

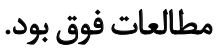

با توجه به ايئكه در نسخه دوم معيار stopp، الب درصد به

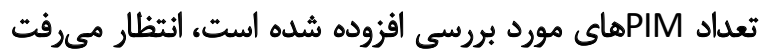

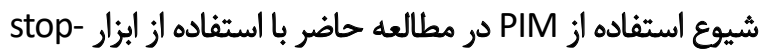

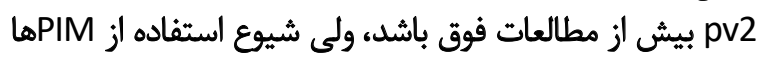

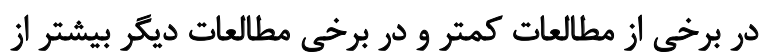
مطالعه حاضر است.

تأزم به نظر مىرسد بايد مطالعات كيفى در مورد عوامل

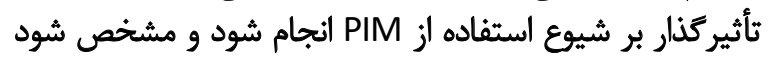

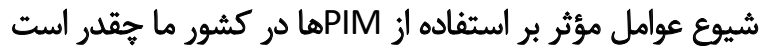

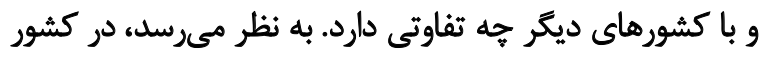

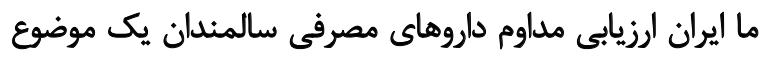
فراموش شده است و در مورد استفاده از داروهاي بارئ بالقوه نامناسب

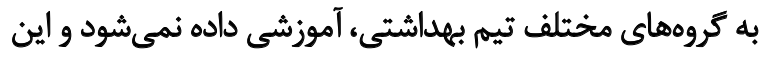

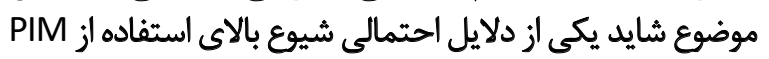

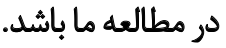

نتايج مقاله مرورى لوجتى و همكارانش نيز نشان داد، بر اساس باس بران

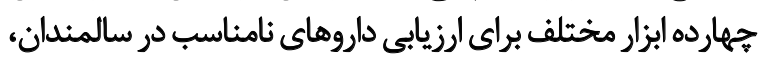

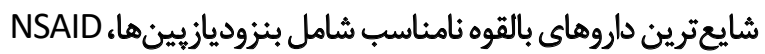

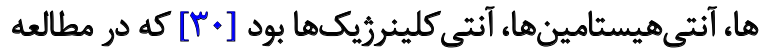

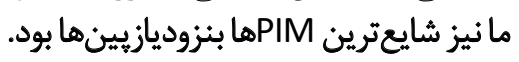

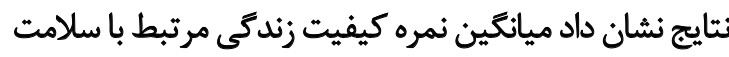

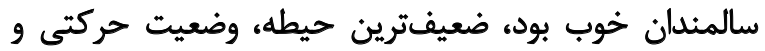
قوى ترين حيطه، وضعيت شنوايى سالمندان بود.آزمون ركان ركرسيون

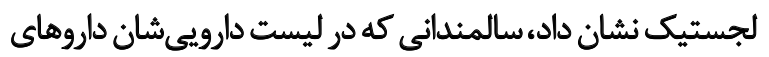

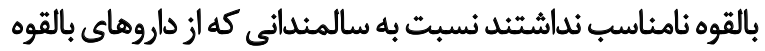

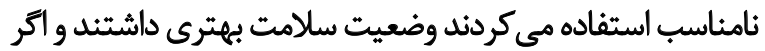

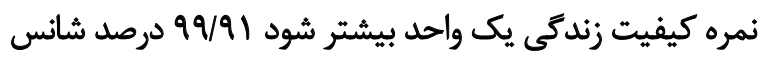

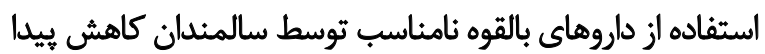


بنزوديازيينها، داروهاى افزايش دهنده خطر اختلالات شناختى و

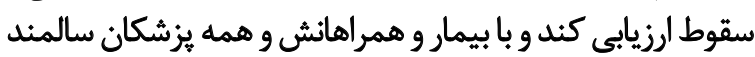

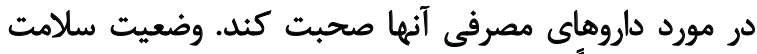

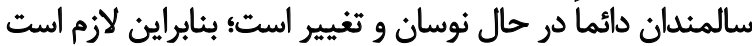
ليست دارويى آنها باطور مستمر ارزيابى شود. يكى از دلايل اشتباهات فراوان در تجويز دارو در سالمندان،

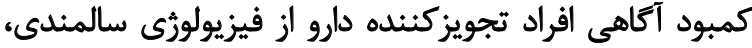

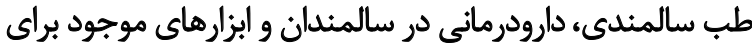

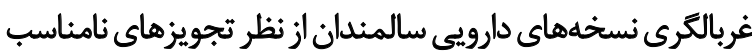

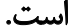

لازم است معرفى ابزارهاى غربالكرى داروهاى سالمندان در

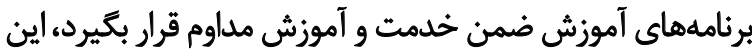

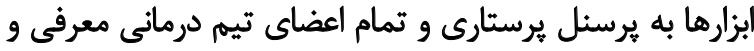

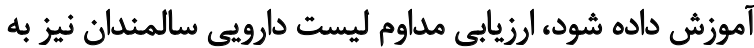

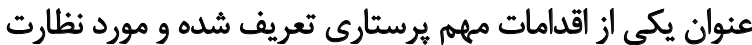

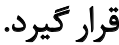

بيشنهاد ميشود به علت اينكه حوادث دارويى ناسازكار

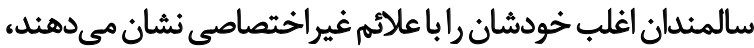

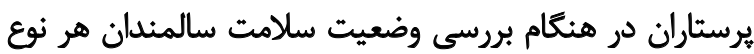

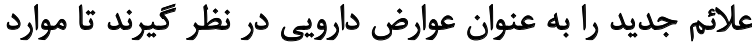

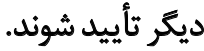

يكى ازمحدوديتهاي مطالعه،عدموجودنسخه هاي الكترونيك ئريك

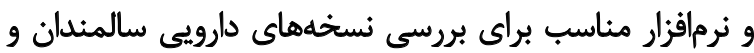

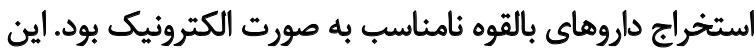

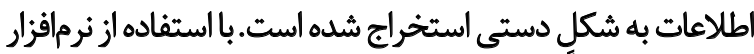

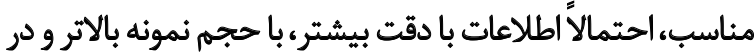

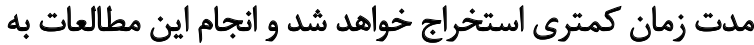

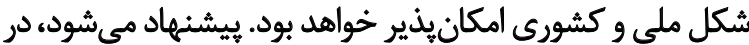

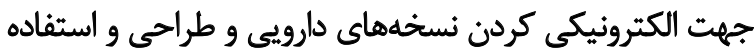

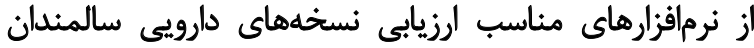

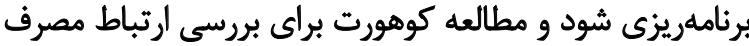

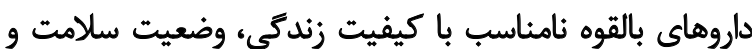

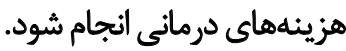

مالاحظات اخلاقى

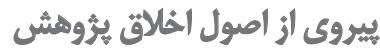

اصول اخلاقي تماهاً در اين مقاله رعايت شده است. شركت

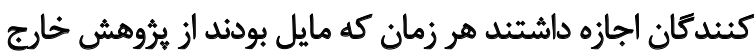

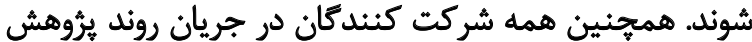

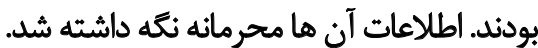

و برخى ديكر كوهورت بودند. به نظر مىرسد اين موضوع به به بهري مطالعات بيشترى نياز دارد.

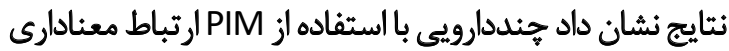

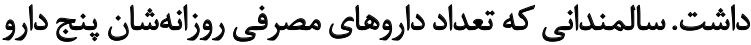

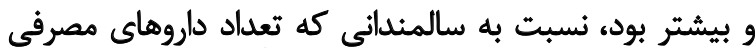

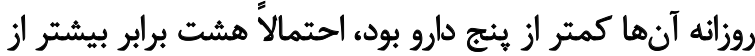
داروهاى بالقوه نامناسب استفاده مى كردند.

نتايج مطالعات بو و همكارانش [1ه]]، ريئوردان و همكارانش

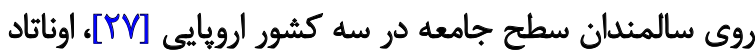

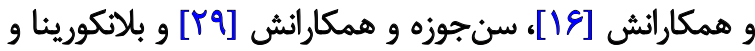

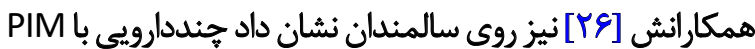

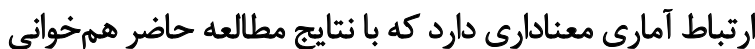
دارد. - ارن

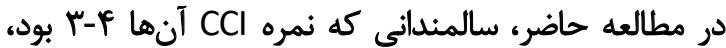

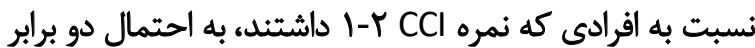

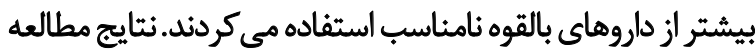

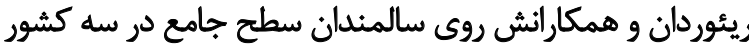

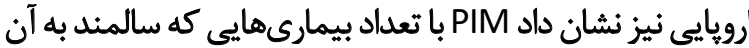

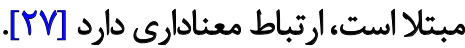
نتايج مطالعه سنجوزه و همكارانش روى سالمندان نشان داد،

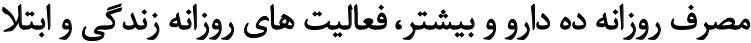

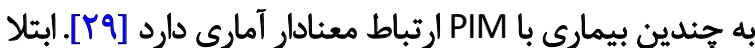

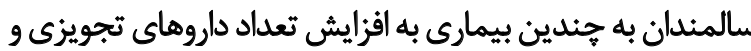

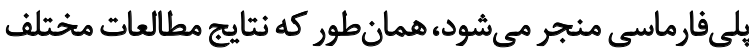

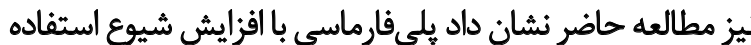

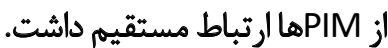

\section{تئيجليَيرى ننهايي}

مصرف داروهاى بالقوه نامناسب در بين سالمندان يك مشكل

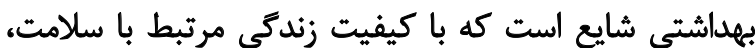

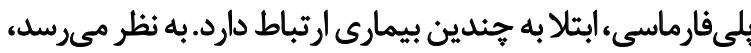

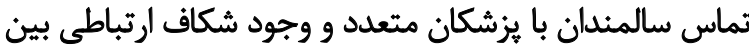

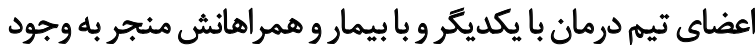
داروهاي بالقوه نامناسب با شيوع بالا در ليست دارويماريى سالمندان

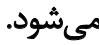

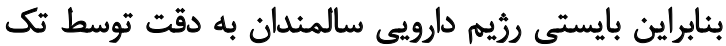

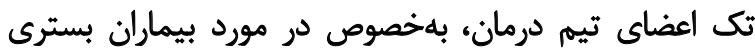

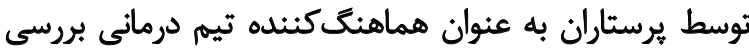

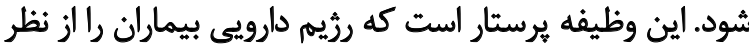

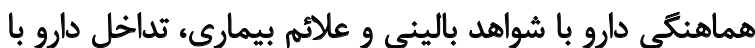

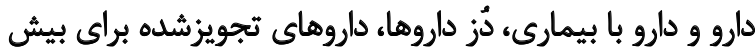

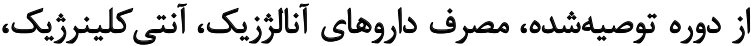




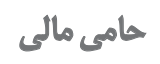

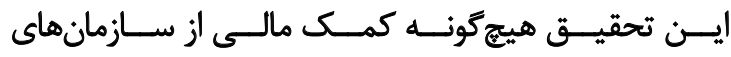

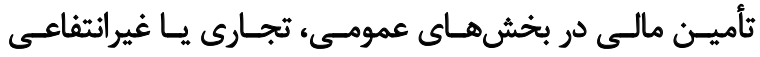

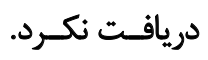

$$
\text { مشار كت نويسيند مان }
$$

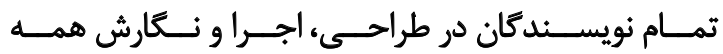

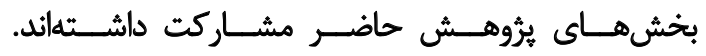

$$
\text { تعارض مناقع }
$$

بنابر اظهار نويسندكان اين مقاله تعارض منافع ندارد. 


\section{References}

[1] Shakeel F, Aamir M, Khan AF, Khan TN, Khan S. Epidemiology of potential drug-drug interactions in elderly population admitted to critical care units of Peshawar, Pakistan. BMC Pharmacology \& Toxicology. 2018; 19:85. [DOI:10.1186/s40360-018-0276-4] [PMID] [PMCID]

[2] van der Stelt CAK, Vermeulen Windsant-van den Tweel AMA, Egberts ACG, van den Bemt PMLA, Leendertse AJ, Hermens WAJJ, et al. The association between potentially inappropriate prescribing and medication-related hospital admissions in older patients: A nested case control study. Drug Safety. 2016; 39(1):7987. [DOI:10.1007/s40264-015-0361-1] [PMID]

[3] Saboor M, Akbari Kamrani AA, Abolfathi Momtaz Y, Sahaf R. Prevalence and associated factors of potentially inappropriate medications among Iranian older adults. Medicinski Glasnik. 2019; 16(1):121-7. [DOI:10.17392/989-19] [PMID]

[4] Canter PH, Ernst E. Herbal supplement use by persons aged over 50 years in Britain: Frequently used herbs, concomitant use of herbs, nutritional supplements and prescription drugs, rate of informing doctors and potential for negative interactions. Drugs \& Aging. 2004; 21(9):597-605. [DOI:10.2165/00002512-20042109000004] [PMID]

[5] Riker GI, Setter SM. Polypharmacy in older adults at home: What it is and what to do about it-implications for home healthcare and hospice, part 2. Home Healthcare Nurse. 2013; 31(2):65-77. [DOI:10.1097/NHH.0b013e31827f43b2] [PMID]

[6] Barry PJ, Gallagher P, Ryan C, O'mahony D. START (Screening Tool to Alert doctors to the Right Treatment) - an evidence-based screening tool to detect prescribing omissions in elderly patients. Age and Ageing. 2007; 36(6):632-8. [DOI:10.1093/ageing/afm118] [PMID]

[7] Gallagher P, Baeyens JP, Topinkova E, Madlova P, Cherubini A, Gasperini B, et al. Inter-rater reliability of STOPP (Screening Tool of Older Persons' Prescriptions) and START (Screening Tool to Alert doctors to Right Treatment) criteria amongst physicians in six European countries. Age and Ageing. 2009; 38(5):603-6. [DOI:10.1093/ageing/afp058] [PMID]

[8] Griebling TL. Re: American Geriatrics Society 2019 updated AGS beers criteria ${ }^{\circledR}$ for potentially inappropriate medication use in older adults. Journal of Urology. 2019; 202(3):438. [DOI:10.1097/ JU.0000000000000409] [PMID]

[9] Zhang S, Meng L, Qiu F, Yang JD, Sun S. Medication-related risk factors associated with health-related quality of life among community-dwelling elderly in China. Patient Preference and Adherence. 2018; 12:529-37. [DOI:10.2147/PPA.S156713] [PMID] [PMCID]

[10] de Lima TJV, Garbin CAS, Garbin AJÍ, Sumida DH, Saliba O. Potentially inappropriate medications used by the elderly: Prevalence and risk factors in Brazilian care homes. BMC Geriatrics. 2013; 13:52. [DOI:10.1186/1471-2318-13-52] [PMID] [PMCID]

[11] Boeker EB, de Boer M, Kiewiet JJS, Lie-A-Huen L, Dijkgraaf MGW, Boermeester MA. Occurrence and preventability of adverse drug events in surgical patients: A systematic review of literature. BMC Health Services Research. 2013; 13:364. [DOI:10.1186/1472-6963-13-364] [PMID] [PMCID]

[12] Curtin D, Gallagher PF, O'Mahony D. Explicit criteria as clinical tools to minimize inappropriate medication use and its consequences. Therapeutic Advances in Drug Safety. 2019; 10 [DOI:10.1177/2042098619829431] [PMID] [PMCID]

[13] Yayla ME, Bilge U, Binen E, Keskin A. The use of START/ STOPP criteria for elderly patients in primary care. The Scientific World Journal. 2013; 2013:165873. [DOI:10.1155/2013/165873 [PMID] [PMCID]

[14] Anrys P, Boland B, Degryse JM, De Lepeleire J, Petrovic M, Marien S, et al. STOPP/START version 2-development of software applications: Easier said than done? Age and Ageing. 2016; 45(5):589-92. [DOI:10.1093/ageing/afw114] [PMID]

[15] Bo M, Gibello M, Brunetti E, Boietti E, Sappa M, Falcone Y, et al. Prevalence and predictors of inappropriate prescribing according to the Screening Tool of Older People's Prescriptions and Screening Tool to Alert to Right Treatment version2 criteria in older patients discharged from geriatric and internal medicine wards: A prospective observational multicenter study. Geriatrics \& Gerontology International. 2019; 19(1):5-11. [DOI:10.1111/ggi.13542] [PMID]

[16] Onatade R, Auyeung V, Scutt G, Fernando J. Potentially inappropriate prescribing in patients on admission and discharge from an older peoples' unit of an acute UK hospital. Drugs \& Ag ing. 2013; 30(9):729-37. [DOI:10.1007/s40266-013-0097-5] [PMID]

[17] Dalleur O, Spinewine A, Henrard S, Losseau C, Speybroeck N Boland B. Inappropriate prescribing and related hospital admissions in frail older persons according to the STOPP and START criteria. Drugs \& Aging. 2012; 29(10):829-37. [DOI:10.1007/ s40266-012-0016-1] [PMID]

[18] Cahir C, Bennett K, Teljeur C, Fahey T. Potentially inappropriate prescribing and adverse health outcomes in community dwelling older patients. British Journal of Clinical Pharmacology. 2014; 77(1):201-10. [DOI:10.1111/bcp.12161] [PMID] [PMCID]

[19] Frély A, Chazard E, Pansu A, Beuscart JB, Puisieux F. Impact of acute geriatric care in elderly patients according to the Screening Tool of Older Persons' Prescriptions/Screening Tool to Alert doctors to Right Treatment criteria in northern France. Geriatrics \& Gerontology International. 2016; 16(2):272-8. [DOI:10.1111/ ggi.12474] [PMID]

[20] Moriarty F, Bennett K, Cahir C, Kenny RA, Fahey T. Potentially inappropriate prescribing according to STOPP and START and adverse outcomes in community-dwelling older people: A prospective cohort study. British Journal of Clinical Pharmacology. 2016; 82(3):849-57. [DOI:10.1111/bcp.12995] [PMID] [PMCID]

[21] Brown JD, Hutchison LC, Li C, Painter JT, Martin BC. Predictive validity of the beers and Screening Tool of Older Persons' Potentially inappropriate prescriptions (STOPP) criteria to detect adverse drug events, hospitalizations, and emergency department visits in the United States. Journal of the American Geriatrics Society. 2016; 64(1):22-30. [DOI:10.1111/jgs.13884] [PMID] [PMCID]

[22] Akkawi ME, Nik Mohamed MH, Md Aris MA. Does inappropriate prescribing affect elderly patients' quality of life? A study from a Malaysian tertiary hospital. Quality of Life Research. 2019; 28(7):1913-20. [DOI:10.1007/s11136-019-02153-5] [PMID]

[23] Glasheen WP, Cordier T, Gumpina R, Haugh G, Davis J, Renda A. Charlson comorbidity index: ICD-9 update and ICD-10 translation. American Health \& Drug Benefits. 2019; 12(4):188-97. [PMID] [PMCID] 
[24] Sintonen H. The 15D instrument of health-related quality of life: Properties and applications. Annals of Medicine. 2001; 33(5):32836. [DOI:10.3109/07853890109002086] [PMID]

[25] Abegaz TM, Birru EM, Mekonnen GB. Potentially inappropriate prescribing in Ethiopian geriatric patients hospitalized with cardiovascular disorders using START/STOPP criteria. PLoS One. 2018; 13(5):e0195949. [DOI:10.1371/journal.pone.0195949] [PMID] [PMCID]

[26] Blanco-Reina E, García-Merino MR, Ocaña-Riola R, AguilarCano L, Valdellós J, Bellido-Estévez I, et al. Assessing potentially inappropriate prescribing in community-dwelling older patients using the updated version of STOPP-START criteria: A comparison of profiles and prevalences with respect to the original version. PLoS One. 2016; 11(12):e0167586. [DOI:10.1371/journal. pone.0167586] [PMID] [PMCID]

[27] Riordan DO, Aubert CE, Walsh KA, Van Dorland A, Rodondi N, Du Puy RS, et al. Prevalence of potentially inappropriate prescribing in a subpopulation of older European clinical trial participants: A cross-sectional study. BMJ Open. 2018; 8(3):e019003. [DOI:10.1136/bmjopen-2017-019003] [PMID] [PMCID]

[28] Formiga F, Vidal X, Agustí A, Chivite D, Rosón B, Barbé J, et al. Inappropriate prescribing in elderly people with diabetes admitted to hospital. Diabetic Medicine. 2016; 33(5):655-62. [DOI:10.1111/dme.12894] [PMID]

[29] San-José A, Agustí A, Vidal X, Formiga F, Gómez-Hernández M, García J, et al. Inappropriate prescribing to the oldest old patients admitted to hospital: Prevalence, most frequently used medicines, and associated factors. BMC Geriatrics. 2015; 15:42. [DOI:10.1186/s12877-015-0038-8] [PMID] [PMCID]

[30] Lucchetti G, Lucchetti ALG. Inappropriate prescribing in older persons: A systematic review of medications available in different criteria. Archives of Gerontology and Geriatrics. 2017; 68:55-61. [DOI:10.1016/j.archger.2016.09.003] [PMID] 